\title{
THE SERVICE PRICING STRATEGIES AND THE STRATEGIC BEHAVIOR OF CUSTOMERS IN AN UNOBSERVABLE MARKOVIAN QUEUE WITH UNRELIABLE SERVER
}

\author{
Kamel MeZiani ${ }^{1, *} \oplus$, Fazia Rahmoune ${ }^{1}$ and Mohammed Said RadjeF ${ }^{1}$
}

\begin{abstract}
A Stackelberg game is used to study the service pricing and the strategic behavior of customers in an unreliable and totally unobservable $M / M / 1$ queue under a reward-cost structure. At the first stage, the server manager, acting as a leader, chooses a service price and, at the second stage, a customer, arriving at the system and acting as a follower, chooses to join the system or an outside opportunity, knowing only the service price imposed by the server manager and the system parameters. We show that the constructed game admits an equilibrium and we give explicit forms of server manager and customers equilibrium behavioral strategies. The results of the proposed model show that the assumption that customers are risk-neutral is fundamental for the standard approach usually used. Moreover, we determine the socially optimal price that maximizes the social welfare and we compare it to the Stackelberg equilibrium. We illustrate, by numerical examples, the effect of some system parameters on the equilibrium service price and the revenue of the server manager.
\end{abstract}

Mathematics Subject Classification. 68M20, 90B22, 91A65.

Received February 9, 2021. Accepted December 14, 2021.

\section{INTRODUCTION}

Nowadays, queueing phenomena can be observed in several fields and in different forms (e.g. supermarkets, hospitals, banks, post offices, fast food restaurants, transportation, telecommunications networks, etc.), and the study of this phenomena has attracted the curiosity of an increasing number of researchers. One area that researchers are currently focusing on in queueing theory is the area of strategic queueing systems. Therefore, a very rich literature is available on this topic. This literature has given many useful ideas in the conception of strategic queueing systems, whose analysis combines two different and important disciplines, it is Queueing Theory and Game Theory. The joint use of these two theories allows the manager of a queueing system to take into consideration and anticipate the strategic reactions of customers to his pricing strategies and to choose the optimal decision (see [8]).

The study of the strategic queueing systems was initiated by Naor [21], who studied the $M / M / 1$ queue with a reward-cost structure, and where the system state is observable. His work was extended in Edelson and

Keywords. Queueing, unreliable server, pricing, strategic behavior, Stackelberg game, equilibrium.

1 Research Unit LaMOS (Modeling and Optimization of Systems), Faculty of Exact Sciences, University of Bejaia, Bejaia 06000, Algeria.

*Corresponding author: kamel.meziani@univ-bejaia.dz

(C) The authors. Published by EDP Sciences, ROADEF, SMAI 2022 
Hildebrand [10] for the unobservable case. Since then, there has been an increasing number of papers that deal with the strategic behavior of customers in different queueing systems. The manuscripts of Hassin and Haviv [13] and Hassin [12] present the main approaches and results on the subject.

The analysis of strategic queueing systems with unreliable server has received a part of attention from researchers because of their interest in the study of the practical problems. In real-world, perfectly reliable servers do not exist. However, in many situations, the server is subject to random failures that affect the system characteristics. Among the works on this topic, we can cite that of Economou and Kanta [9], who extended the model of Naor [21], including the failures and the repairs periods of the server, while considering the totally observable case and the almost observable case. Later, Li et al. [17] enhanced this work by dealing with two other types of information: the almost unobservable case and the totally unobservable case. Wang and Zhang [26] generalized the model studied in [9] by considering an $M / M / 1$ queueing system with an unreliable server and delayed repairs. The failed server experiences a delayed time exponentially distributed before the start of the repair process. Yu et al. [28] completed the model of Wang and Zhang [26] in the almost unobservable case and then in the totally unobservable case. Li et al. [16] analyzed the customers behavior in a Markovian server queue with partial breakdowns. So, during the failure period, customers are served at a low rate compared to the service rate when the system is operational. Two cases are considered: totally observable and totally unobservable. This work [16] was extended by $\mathrm{Yu}$ et al. [29] to the almost observable and almost unobservable cases. Boudali and Economou [3] studied the behavior of customers who decide whether to join or not a Markovian queueing system with catastrophes. When the server fails, all customers are forced to leave the system. Two cases are analyzed: the totally observable and the totally unobservable. The same model, discussed in [3], was then examined by Boudali and Economou [4], where they added the assumption that arriving customers are allowed to join the system even if they find the server unavailable. Tian and Wang [23] treated this model of Boudali and Economou [4] in the almost unobservable case. Chen and Zhou [7] studied the customers behaviors in a Markovian queue with setup time and breakdowns. The server is turned off each time the system becomes empty, and it begins an exponential installation time when a customer arrives at the system. When the server fails, a repair process is launched immediately and no customer is accepted during the failure and the repair periods of the server. In this work, three cases are considered: totally observable, almost unobservable and totally unobservable. For recent works in this topic, we recommend the following works: Yu et al. [30], Tian and Wang [24], Gao et al. [11], Aghsami and Jolai [1], Li and Wang [15], Ma and Zhang [18], Zhang and Xu [33], Zhang and Wang [32].

Most of the works mentioned so far consider a game among customers. Recently, a different approach has emerged in the strategic queueing works. The latter is represented by a dynamic game between the different agents (customers, server, social optimizer, ... ) intervening in the system. In some of these works, authors seek to determine the optimal price of service to be offered by the system according to the strategic behavior of the customers. Chen and Frank [5] adapted the classic model of Naor [21], where the server can adjust his service price according to the current state of the queue. At their arrival times, customers observe the state of the queue and the service price, and then decide to join the system or not. In this model, a customer who does not join the system will have a fixed reward. Much later, Chen and Frank [6] completed this work in the unobservable case. Manou et al. [19] studied the pricing problem for a transportation station, using the queueing theory and a two-stage game among the customers and the administrator, where customer strategies depend on the level of information on the queue (totally observable, totally unobservable or almost observable). Jagannathan et al. [14] investigated a decision-making process between customers and two servers in a cognitive radio network, where a Markovian queueing system is considered with the server 2. The server 1 can adjust its service price first in order to maximize its revenue. Then, customers have the opportunity to buy a service from server 1 or enjoy a free service from server 2 that can be interrupted by random breakdowns. Upon their arrival, customers observe the price imposed by the server 1 and the state of the server 2, but they do not have information on the number of customers in the queue facing the server 2, then they decide between using server 1 or using server 2 . Wang and Zhang [27] contributed to the analysis of a Markovian retrial queue and delayed vacations for Local Area Network applications in which the server will take delayed vacations at the end of the service. For LAN applications, they did not only study the strategic behavior of customers but also considered the strategic monopoly (service 
provider). They modeled this situation as a two-stage game (Stackelberg competition) between the service provider and the customers. At the first stage, the service provider sets a service price first, which is known by all customers before they act. Then, at the second stage, based on the value of the price charged, the customers make their decisions to balk or to enter the system for service based on their expected payoff and available information about the status of the server. A Closely related work to the model analyzed in this paper was treated by Meziani et al. [20]. In that work, they investigated the service pricing and the strategic behavior of customers as a Stackelberg game (the server acts first as the leader and the customers as the followers), and the social welfare in a totally unobservable $M / M / 1$ queue system with unreliable server and delayed repairs (when a failure occurs, the server becomes unavailable and waits for a random time to start the repair process).

As we mentioned above, there are many works that have considered the server breakdown problem and others that have considered the service pricing problem in the study of the Markovian queueing systems with strategic customers. Our work combines both these problems by considering the pricing of the service provided in an $M / M / 1$ queueing system with an unreliable server and strategic customers where the system state is totally unobservable. The strategic interactions are described as Stackelberg game, where the server acts as the leader and a potential customer as the follower. We show that the constructed game admits a Stackelberg equilibrium and we give explicitly the equilibrium strategies of the server and the customers. Contrary to the standard approach, usually used in strategic queueing system, where customers are assumed to have a "risk-neutral" profile (see [12]), we assume in the approach developed in this paper that customers have an optimizer profile, in the sense that a customer will choose the decision that will optimize his utility. We further study how the social planner determines the price to charge a joining customer. We synthesize the results in the form of two decision-making algorithms: the first one for the choice of the equilibrium service price and the second one for the choice of social service price. These two algorithms aim to describe the process that the service manager or the administrator should follow to determine the service price to apply by the server. Finally, we illustrate via numerical examples the impact of the service price selected by the server on customers' decisions and the consequences of the customer's decisions on the server's decision. This model can be considered as a development and a combination of the models of Li et al. [17] and Chen and Frank [6]. Furthermore, the model examined in this paper can be viewed also as a special case of the model of Meziani et al. [20], where in our model the repairs are immediate, and in the model of Meziani et al. [20] the repairs are delayed.

This work is of a theoretical and practical interest. Indeed, the model under consideration has broad applications in many fields, and can be adapted for many real-situations, namely: the call centers [17], cognitive radio network [14], transportation [19], manufacturing industries [6,16,26], supply-chain systems [28]... etc. However, the example of made-to-order systems given by Meziani et al. [20] can be also used as an application for our model. In addition, one of the many real-life situations to which our model can be applied is the following situation: Suppose a customer goes to an online store to purchase a product or a service. Similar to a real store, this type of virtual store displays the price and delivery time information about the product or the service. However, the delivery may be interrupted by unexpected circumstances (which occur randomly). After receiving the price and delivery information, a customer, upon arrival, has the choice to order the product or the service online or to buy it elsewhere (in another virtual or real store).

The remainder of this paper is structured as follows. Section 2 presents the description of the unreliable $M / M / 1$ system, under a reward-cost structure, while the Section 3 presents the main characteristics of the considered queueing system. In Section 4, we model the considered problem as Stackelberg game. Section 5 examines customers equilibrium strategies and the problem of maximizing server manager revenue. We give an example in Remark 5.3, showing that the assumption that all customers have a "risk-neutral" profile must be assumed in the standard approach, otherwise, a customer with an optimizer profile might find it advantageous to deviate from the equilibrium strategy (obtained by the standard approach). Then, we consider the social welfare problem in Section 6 and some numerical illustrations are presented in Section 7, where we analyze the effect of the system parameters on the joining probability of customers, the service price and the revenue of the server manager. We provide a conclusion and some remarks in Section 8. Finally, we end with an appendix that contains the proofs of the different obtained results and the two decision-making algorithms in Appendix A. 


\section{MODEL DESCRIPTION}

We consider a Markovian $M / M / 1$ queue with infinite capacity and unreliable server in which customers arrive according to a Poisson process with rate $\lambda$. The service times are assumed to be exponentially distributed random variables with rate $\mu$ and the service is performed according to FCFS policy. The server is subject to random breakdowns following a Poisson process with parameter $\theta$. When a failure occurs, the server becomes inactive, and its repair is immediately started with an exponential distribution of rate $r$. We assume that inter-arrival times, service times, inter-failure times, and repair times are mutually independent. This system is described by the process $\{N(t), I(t), t \geq 0\}$, where $N(t)$ represents the number of customers in the system at time $t$, and $I(t)$ represents the server state (operational or breakdown) at time $t$.

Furthermore, we assume that there is a reward-cost structure that reflects the desire of customers for the service to be acquired and their unwillingness for waiting. A customer will pay a price $P$ (service price) fixed by the server manager, receives a reward of $R$ units (service value) if the service is performed and loses a waiting $\operatorname{cost} C$ per unit of time for the time remaining in the system. We also associate a fixed reward, noted by $v$, which represents the reward of a customer, who decides to choose an outside opportunity. This outside opportunity may reflect a customer's satisfaction without being served, it may also reflect a customer's gain if it is served elsewhere.

The server manager can choose a price $P$, allowing him to maximize his revenue and assuming that the customers have an optimizer profile. Upon his arrival, a customer has the opportunity to join the system or enjoy an outside opportunity, this decision once taken is assumed irrevocable. Customers are assumed to have an optimizer profile, in the sense they make their decisions with the aim of maximizing their utility functions. Their decision-making process will be described in Section 4 . The system parameters $(\lambda, \mu, \theta, r)$ and the economic parameters $(R, P, C, v)$ are common knowledge for the server manager and the customers. However, when a customer has to make his decision, he does not observe the actual length of the queue and the state of the server.

We will study the decision process of the server manager and customers, and we will determine the strategic behavior corresponding to their objectives. We begin by determining the characteristics of the system that will be useful to define server and customers utility functions.

\section{SySTEM CHARACTERISTICS}

We considered a Markovian $M / M / 1$ unreliable queue, whose the state of the system at time $t$ is characterized by the pair $(N(t), I(t))$, where $N(t)$ represents the number of customers in the system and $I(t)$ the state of the server at time $t$, which takes " 1 " if the server is operational and " 0 " in the case of breakdown. We consider that the system is totally unobservable by the customer at his arrival time. Since customers are homogeneous, we assume that each arriving customer joins the system with a probability $q$, generating a joining rate $\lambda_{e}=\lambda q$.

The stochastic process $\{(N(t), I(t)): t \geq 0\}$ is a continuous time Markov chain with state space $S=$ $\{(n, 1), n \geq 0\} \cup\{(n, 0), n \geq 0\}$, and the transition diagram associated with it is given by Figure 1 .

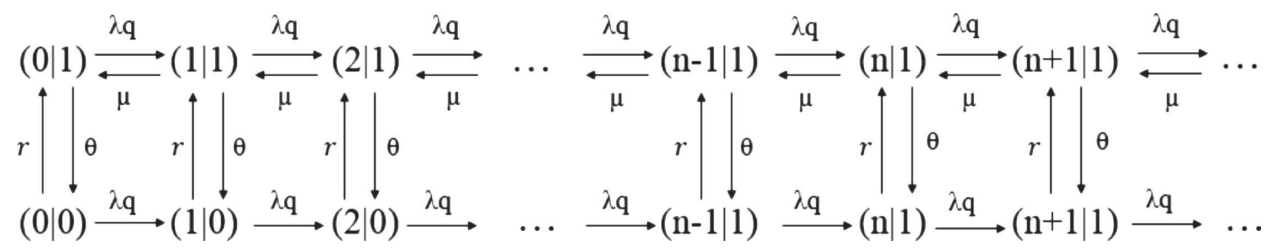

FIGURE 1. The transition diagram. 
The balance equations for the stationary distribution of the Markov chain $\{(N(t), I(t)): t \geq 0\}$ are given as follows:

$$
\begin{array}{rlrl}
(\lambda q+\theta) P_{01} & =\mu P_{11}+r P_{00} ; \\
(\lambda q+\theta+\mu) P_{j 1} & =\mu P_{j+11}+\lambda q P_{j-11}+r P_{j 0}, \quad & & (j \geq 1) ; \\
(\lambda q+r) P_{00} & =\theta P_{01} ; & & (j \geq 1) ; \\
(\lambda q+r) P_{j 0} & =\theta P_{j 1}+\lambda q P_{j-10}, &
\end{array}
$$

where, $P_{j i}=P[N(t)=j, I(t)=i], \forall t \geq 0, \forall j \geq 0$ and $\forall i=\{0,1\}$ are the system state probabilities.

Using these equations and the stability condition $\lambda(r+\theta)<r \mu$, we obtain the expression of the customer's average sojourn time in the system $\overline{T_{s}}$, which is given as follows:

$$
\overline{T_{s}}(q)=\frac{\mu \theta+(r+\theta)^{2}}{(r+\theta)(r \mu-\lambda q(r+\theta))} .
$$

This characteristic will be useful for the definition of some quantities related to the game in the next section.

\section{Modeling as a Stackelberg game}

The problem treated in this paper concerns a server manager, desiring to set the same service price $P$ to all customers, taking into consideration that his server may experience failures, which lead to the interruption of the service or the slowing down of the service rate. We assume that customers arrive sequentially at the server and the manager adopts a policy of communication with the customers upon their arrival in the system before they make their decision to join the system or to choose an outside opportunity. In our study case, upon their arrival at the system, a customer:

(1) is informed about:

(a) the server's decision (the service price);

(b) system parameters $(\lambda, \mu, \theta, r)$ and the economic parameters $(R, C, v)$.

(2) is not informed about:

(a) the decisions made by customers who arrived before him;

(b) his order of arrival in the system, and therefore on the number of customers who arrived before him in the system;

(c) the number of customers in the queue;

(d) the state of the server (operational or breakdown).

In the literature on strategic queueing systems, there are several works that model similar situations in the form of two-stage models. At the first stage, the server manager's objective is to set the service price, maximizing his profit, which also depends on the customers' decisions. At the second stage, the strategic interactions of customers are described by a non-standard hierarchical game with imperfect and incomplete information, as, among others, the number of players (customers) is unknown.

To solve this game, the concept of symmetric equilibrium is used, based on the assumed assumption that the players are homogeneous. For the calculation of this equilibrium, it is further assumed that customers decide to join as soon as their utility is greater than or equal to zero (respectively to the utility associated with choosing an outside opportunity when it exists). As this hypothesis, on the behavior of customers, has a direct influence on the function of the best responses of the customers, in relation to the price set by the server, and therefore on the equilibrium of the game, we took the initiative to designate that the customers have a neutral risk profile, a term used in the classic approach (see Hassin and Haviv [13] and a detailed description of the resolution method for this type of game between customers can be found in the chapter of [8]).

In this paper, we propose another two-stage model, and we describe the methodology of its formulation as follows. At the first stage, the server manager, acting as a leader, chooses the service price that he offers to the 


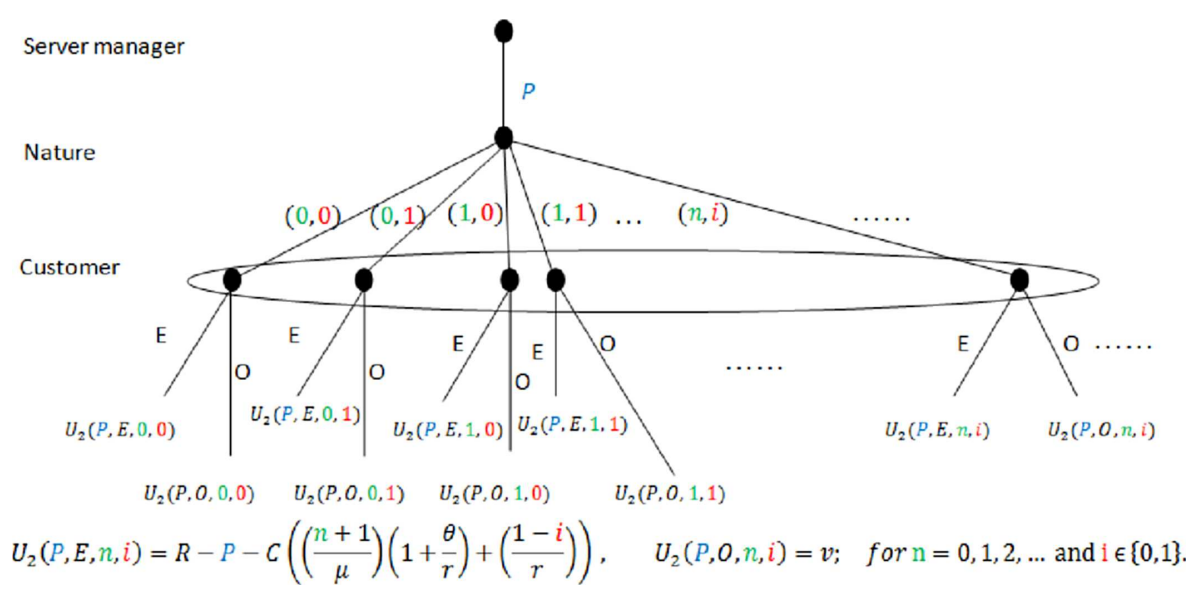

FIGURE 2. Decision tree of a customer upon arrival at the server.

arriving customers in his system. His objective is to set a service price, maximizing his utility, depending on the number of customers joining his service.

Customers arrive sequentially at the server. Each customer must decide whether to join the service or to choose an outside opportunity, based on the information available to him (the service price chosen by the manager and the system characteristics (the service value, the outside opportunity value, the sojourn time in the queue, the server failure frequency, the repair rate, ...)) and in the absence of information about the order of his arrival at the service, the state of the server, the number of customers in the queue, the decisions chosen by the customers who arrived before him.

Since the service manager applies the same service price to all customers, then each customer, arriving in the system, must make his decision on the basis of the same information (the same service price). In other words, each customer is called to solve an uncertain decision making problem, illustrated by Figure 2, where $n \in \mathbb{N}$ represents the number of customers in the system and the state of the server, which takes " $i=1$ ", if the server is operational and " $i=0$ " in the case of breakdown. The term "game with chance moves" is used in Basar and Olsder [2], to denote the game of Figure 2 between the server manager and a potential customer.

Thus, if a customer comes to the server and makes the decision to join the system with probability $q$ and to choose the outside opportunity with probability $(1-q)$, then his expected utility is:

$$
U_{2}(P, q)=\sum_{n=0}^{\infty}\left[U_{2}(P, E, n, 1) q+U_{2}(P, O, n, 1)(1-q)\right] P_{n 1}+\sum_{n=0}^{\infty}\left[U_{2}(P, E, n, 0) q+U_{2}(P, O, n, 0)(1-q)\right] P_{n 0},
$$

where $P$ denotes the service price, $E$ the decision of a customer to join the system, $O$ the decision of a customer for the outside opportunity. $P_{n i}$ are the system state probabilities with $n$ representing the number of customers in the queue and $i$ representing the server state.

The utility of a customer who finds " $n$ " customers in the system and the server state is " $i$ " and decides to join the system, is given by:

$$
U_{2}(P, E, n, i)=R-P-C\left(\frac{n+1}{\mu}\right)\left(1+\frac{\theta}{r}\right)\left(\frac{1-i}{r}\right), \quad \forall i \in\{0,1\} \text { and } n \in \mathbb{N},
$$

and the utility of a customer who finds " $n$ " customers in the system and the server state is " $i$ " and decides to join the outside opportunity, is given by:

$$
U_{2}(P, O, n, i)=v, \quad \forall i \in\{0,1\} \text { and } n \in \mathbb{N} .
$$


Hence,

$$
\begin{aligned}
U_{2}(P, q)= & \sum_{n=0}^{\infty}\left[U_{2}(P, E, n, 1) P_{n 1}+U_{2}(P, E, n, 0) P_{n 0}\right] q+\sum_{n=0}^{\infty}\left[U_{2}(P, O, n, 1) P_{n 1}+U_{2}(P, O, n, 0) P_{n 0}\right](1-q), \\
= & q \sum_{n=0}^{\infty}\left[U_{2}(P, E, n, 1) P_{n 1}+U_{2}(P, E, n, 0) P_{n 0}\right]+(1-q) \sum_{n=0}^{\infty}\left[U_{2}(P, O, n, 1) P_{n 1}+U_{2}(P, O, n, 0) P_{n 0}\right], \\
= & q \sum_{n=0}^{\infty}\left[\left(R-P-C\left(\frac{n+1}{\mu}\right)\left(1+\frac{\theta}{r}\right)\right) P_{n 1}\right. \\
& \left.+\left(R-P-C\left(\left(\frac{n+1}{\mu}\right)\left(1+\frac{\theta}{r}\right)+\frac{1}{r}\right)\right) P_{n 0}\right]+(1-q) \sum_{n=0}^{\infty}\left[v\left(P_{n 1}+P_{n 0}\right)\right], \\
= & q \sum_{n=0}^{\infty}\left[\left(R-P-C\left(\frac{n+1}{\mu}\right)\left(1+\frac{\theta}{r}\right)\right)\left(P_{n 1}+P_{n 0}\right)-\frac{C}{r} P_{n 0}\right]+(1-q) v, \\
= & \left.q\left(R-P-C\left(\frac{\sum_{n=0}^{\infty} n P_{n}+1}{\mu}\right)\left(1+\frac{\theta}{r}\right)\right]-\frac{C}{r} \sum_{n=0}^{\infty} P_{n 0}\right]+(1-q) v, \\
= & \left.q\left(R-P-C\left(\frac{\frac{\lambda q\left(\mu \theta+(r+\theta)^{2}\right)}{(r+\theta)(r \mu-\lambda q(r+\theta))}+1}{\mu}\right)\left(1+\frac{\theta}{r}\right)\right)-\left(\frac{C}{r}\right)\left(\frac{\theta}{r+\theta}\right)\right]+(1-q) v, \\
= & q\left(R-P-C\left(\frac{\left(\mu \theta+(r+\theta)^{2}\right)}{(r+\theta)(r \mu-\lambda q(r+\theta))}\right)+(1-q) v,\right. \\
U_{2}(P, q)= & q\left(R-P-C . T_{s}(q)\right)+(1-q) v, \\
& {[(}
\end{aligned}
$$

where $R$ is the reward (value of the service) per unit of time, $C$ is the waiting cost per unit of time, $v$ is the reward associated to the outside opportunity and $\overline{T_{s}}(q)$ is the customer's average sojourn time in the system, given by (3.1).

Assuming that customers are rational players, as is generally believed in classical game theory, each player would adopt the mixed strategy that maximizes his expected utility function $U_{2}$, which is the same for all players (customers).

In this case, for a service price $P$ and for a joining probability $q$ of a customer, the manager's expected payoff will be:

$$
U_{1}(P, q)=\lambda q P .
$$

For the server manager, the customers are considered homogeneous, since they have the same utility function $U_{2}(P, q)$, and they have an optimizer profile (i.e. the customers seek to maximize their utility in the face of the price imposed by the server manager), the reaction of each customer will be to determine the joining probability $q$ maximizing his utility. Having considered that the manager will apply the same price for all his customers, the problem can be modeled as a two-player Stackelberg game.

The server manager, acting as the leader, chooses his service price, maximizing his expected utility

$$
\max _{P \in[0,+\infty)} U_{1}(P, q) .
$$

The potential customer (the follower player) chooses his mixed strategy $(q, 1-q) \in[0,1]^{2}$ maximizing his expected utility:

$$
\max _{q \in[0,1]} U_{2}(P, q),
$$


where $U_{2}(P, q)=q\left(R-P-C\left(\frac{\mu \theta+(r+\theta)^{2}}{(r+\theta)(r \mu-\lambda q(r+\theta))}\right)\right)+(1-q) v$.

Considering that each customer, arriving at the system and finding a service price $P$, chooses his decision to join the system with the probability $q \in[0,1]$, maximizing his expected utility $U_{2}(P, q)$, i.e. the solution of problem (4.2). Since this problem has a unique solution (see Lem. 5.1), then all customers will choose the same mixed strategy.

In order to distinguish this approach from the standard one, where customers choose the mixed equilibrium strategy of a non-standard game, computed with the risk-neutral assumption, we have taken the initiative to consider in our approach that customers have an optimizer profile, with reference to the optimization problem (4.2) that determines the mixed strategy to be chosen.

In Section 5, we illustrate, through an example, the importance of specifying the customers' profile.

\section{The GAME RESOlution}

The backward induction method is adopted to solve the Stackelberg game. We start by solving the last stage of the game (customers behavior) for a price $P$ set by the server manager. The obtained solution will be injected into the server manager utility function of the first stage to determine the equilibrium service price.

\subsection{Study of the second stage of the game}

In this second stage of the game, we study a customer's strategic behavior for a service price $P$. The customer's objective is to determine his best response $\mathrm{BR}_{C}(P)$ to the price $P$ imposed by the server manager. In other words, the customer should solve the problem:

$$
\operatorname{BR}_{C}(P)=q^{*}(P)=\arg \max _{q \in[0,1]} U_{2}(P, q),
$$

which would lead him to adopt a mixed strategy $\alpha^{*}(P)=\left(q^{*}(P), 1-q^{*}(P)\right)$, i.e. join the system with a probability $q^{*}(P)$ or choose an outside opportunity with a probability $1-q^{*}(P)$.

We assume that the server manager has set a service price $P$ and we consider the arrival of a customer to the system.

To facilitate the reading of the paper, we will adopt the following notations which depend only on the system parameters:

$$
A=R-v-C\left(\frac{\mu r\left(\mu \theta+(r+\theta)^{2}\right)}{(r+\theta)(r \mu-\lambda(r+\theta))^{2}}\right), \quad \text { and } \quad B=R-v-C\left(\frac{\mu \theta+(r+\theta)^{2}}{r \mu(r+\theta)}\right) .
$$

Considering the condition stability $r \mu>\lambda(r+\theta)$, we deduce the relation $r \mu>\lambda q(r+\theta)$ and, then, the inequality: $A<B$.

Since $A$ and $B$ depend only on the system parameters, then the values of $A$ and $B$ can have different signs. Indeed, if for example the value of the outside opportunity $v$ or/and the waiting cost $C$ are more important than the value of the service $R$, we will have $B \leq 0$. In the opposite case, i.e. if the value of the service $R$ is more attractive than the value proposed by the outside opportunity $v$ or/and the waiting $\operatorname{cost} C$, we can have the cases where $A \geq 0$ (which implies $B>0$ ) or $B>0$ and $A \leq 0$. These different cases conduce to important results, such as the ones related to the Stackelberg equilibrium and the socially optimal price (see Sects. 5 and 6 respectively).

The following lemma gives us the explicit forms of the best response of a customer arriving at the system and informed about the price $P$ chosen by the server manager.

Lemma 5.1. In the totally unobservable $M / M / 1$ queue with an unreliable server, there exists a unique best response $\mathrm{BR}_{C}(P)$, for a customer arriving at the system and informed about the price $P \geq 0$ chosen by the 
server manager, which takes the form:

$$
\operatorname{BR}_{C}(P)=q^{*}(P)= \begin{cases}0, & \text { if } P \geq R-v ; \\ 0, & \text { if } P<R-v, B \leq 0 ; \\ 1, & \text { if } P<R-v, B>0, A \geq 0, P \in[0, A] ; \\ \frac{1}{\lambda}\left(\frac{\mu r}{r+\theta}-\sqrt{\left.\frac{C \mu r\left(\mu \theta+(r+\theta)^{2}\right)}{(r+\theta)^{3}(R-P-v)}\right),},\right. & \text { if } P<R-v, B>0, A \geq 0, P \in[A, B] ; \\ \frac{1}{\lambda}\left(\frac{\mu r}{r+\theta}-\sqrt{\left.\frac{C \mu r\left(\mu \theta+(r+\theta)^{2}\right)}{(r+\theta)^{3}(R-P-v)}\right),},\right. & \text { if } P<R-v, B>0, A \leq 0, P \in[0, B] ; \\ 0, & \text { if } P<R-v, B>0, P \geq B,\end{cases}
$$

where $q^{*}(P)$ is the probability that the customer decides to join, knowing the fixed price $P$.

The expected utility of the customer will take the form:

$$
U_{2}\left(P, q^{*}(P)\right)= \begin{cases}v, & \text { if } P \geq R-v ; \\ v, & \text { if } P<R-v, B \leq 0 ; \\ R-P-C\left(\frac{\mu \theta+(r+\theta)^{2}}{(r+\theta)(\mu r-\lambda(r+\theta))}\right), & \text { if } P<R-v, B>0, A \geq 0, P \in[0, A] ; \\ \widetilde{U}_{2}\left(P, q^{*}(P)\right), & \text { if } P<R-v, B>0, A \geq 0, P \in[A, B] ; \\ \widetilde{U}_{2}\left(P, q^{*}(P)\right), & \text { if } P<R-v, B>0, A \leq 0, P \in[0, B] ; \\ v, & \text { if } P<R-v, B>0, P \geq B,\end{cases}
$$

where $\widetilde{U}_{2}\left(P, q^{*}(P)\right)=\left(R-P-C\left(\frac{\mu \theta+(r+\theta)^{2}}{(r+\theta)\left(\mu r-\lambda q^{*}(P)(r+\theta)\right)}\right)\right) q^{*}(P)+v\left(1-q^{*}(P)\right)$.

Proof. The proof of the Lemma 5.1 is provided in Appendix A.1.

The Lemma 5.1 describes the strategic behavior of a potential customer, arriving at the system, according to the price displayed by the server manager.

\subsection{Study of the first stage of the game}

Anticipating the strategic behavior of the customers $\mathrm{BR}_{C}(P)=q^{*}(P)$ according to the price $P$ fixed by the server manager, the utility of the server manager will take the form:

$$
U_{1}(P)=U_{1}\left(P, q^{*}(P)\right)= \begin{cases}0, & \text { if } P \geq R-v ; \\ 0, & \text { if } P<R-v, B \leq 0 ; \\ \lambda P, & \text { if } P<R-v, B>0, A \geq 0, P \in[0, A] ; \\ \left(\frac{\mu r}{r+\theta}-\sqrt{\frac{C \mu r\left(\mu \theta+(r+\theta)^{2}\right)}{(r+\theta)^{3}(R-P-v)}}\right) P, & \text { if } P<R-v, B>0, A \geq 0, P \in[A, B] ; \\ \left(\frac{\mu r}{r+\theta}-\sqrt{\left.\frac{C \mu r\left(\mu \theta+(r+\theta)^{2}\right)}{(r+\theta)^{3}(R-P-v)}\right) P,},\right. & \text { if } P<R-v, B>0, A \leq 0, P \in[0, B] ; \\ 0, & \text { if } P<R-v, B>0, P \geq B .\end{cases}
$$

Based on the system parameters determining the values of $A, B$ and the price $P$ set by the server manager, the relation (5.2) determines the joining probability of customers and the relation (5.3) determines the corresponding server manager utility.

The objective of the server manager is to choose a service price maximizing his revenue. Having defined the strategic behavior of the customers $\mathrm{BR}_{C}(P)=q^{*}(P)$ and according to the service price $P$ which he chooses, the server manager is expected to resolve the problem:

$$
U_{1}(P)=U_{1}\left(P, q^{*}(P)\right) \longrightarrow \max _{P \geq 0} .
$$


To facilitate the follow-up of the next reasonings for solving the problem (5.4), we introduce the notations:

$$
F=\frac{L+K^{1 / 3}+\left(\frac{L(L+24 D(R-v))}{K^{1 / 3}}\right)}{12 D},
$$

where

$$
\left\{\begin{array}{l}
L=C\left(\mu \theta+(r+\theta)^{2}\right), \\
D=\mu r(r+\theta), \text { and } \\
K=L\left(L^{2}+36 D L(R-v)+216 D^{2}(R-v)^{2}\right)+24 \sqrt{3} \sqrt{L^{2} D^{3}(L+27 D(R-v))(R-v)^{3}} .
\end{array}\right.
$$

Theorem 5.2. The Stackelberg game, modeling the strategic behavior of the server manager and customers in a totally unobservable $M / M / 1$ queue with an unreliable server, admits an equilibrium $\left(P^{*}, \alpha^{*}=\left(q^{*}(P), 1-\right.\right.$ $\left.\left.q^{*}(P)\right)\right) \in[0, \infty) \times[0,1]^{2}$, where:

$$
P^{*}= \begin{cases}{[0,+\infty),} & \text { if } B \leq 0 \\ A, & \text { if } B>0, A \geq 0 \text { and } 0 \leq P^{m} \leq A \\ P^{m}, & \text { if } B>0, A \geq 0 \text { and } A \leq P^{m} \leq B \\ P^{m}, & \text { if } B>0 \text { and } A \leq 0\end{cases}
$$

and

$$
\alpha^{*}= \begin{cases}(0,1), & \text { if } B \leq 0 \\ (1,0), & \text { if } B>0, A \geq 0 \text { and } 0 \leq P^{m} \leq A \\ \left(q^{*}\left(P^{m}\right), 1-q^{*}\left(P^{m}\right)\right), & \text { if } B>0, A \geq 0 \text { and } A \leq P^{m} \leq B \\ \left(q^{*}\left(P^{m}\right), 1-q^{*}\left(P^{m}\right)\right), & \text { if } B>0 \text { and } A \leq 0\end{cases}
$$

where

$$
P^{m}=R-v-F, \quad \text { and } \quad q^{*}\left(P^{m}\right)=\frac{1}{\lambda}\left(\frac{\mu r}{r+\theta}-\sqrt{\frac{C \mu r\left(\mu \theta+(r+\theta)^{2}\right)}{(r+\theta)^{3}\left(R-P^{m}-v\right)}}\right) .
$$

The expected revenue of the server manager at the Stackelberg equilibrium is:

$$
U_{1}^{*}\left(P^{*}, q^{*}\right)= \begin{cases}0, & \text { if } B \leq 0 ; \\ \lambda\left(R-v-C\left(\frac{\mu r\left(\mu \theta+(r+\theta)^{2}\right)}{(r+\theta)(r \mu-\lambda(r+\theta))^{2}}\right)\right), & \text { if } B>0, A \geq 0 \text { and } 0 \leq P^{m} \leq A ; \\ \left(\frac{\mu r}{r+\theta}-\sqrt{\frac{C \mu r\left(\mu \theta+(r+\theta)^{2}\right)}{(r+\theta)^{3}\left(R-P^{m}-v\right)}}\right) P^{m}, & \text { if } B>0, A \geq 0 \text { and } A \leq P^{m} \leq B ; \\ \left(\frac{\mu r}{r+\theta}-\sqrt{\frac{C \mu r\left(\mu \theta+(r+\theta)^{2}\right)}{(r+\theta)^{3}\left(R-P^{m}-v\right)}}\right) P^{m}, & \text { if } B>0 \text { and } A \leq 0 .\end{cases}
$$

The expected utility of a customer at the Stackelberg equilibrium is given by:

$$
U_{2}^{*}\left(P^{*}, q^{*}\right)= \begin{cases}v, & \text { if } B \leq 0 ; \\ R-A-C\left(\frac{\mu \theta+(r+\theta)^{2}}{(r+\theta)(r \mu-\lambda(r+\theta))}\right), & \text { if } B>0, A \geq 0 \text { and } 0 \leq P^{m} \leq A ; \\ U_{2}\left(P^{m}, q^{*}\left(P^{m}\right)\right), & \text { if } B>0, A \geq 0 \text { and } A \leq P^{m} \leq B ; \\ U_{2}\left(P^{m}, q^{*}\left(P^{m}\right)\right), & \text { if } B>0 \text { and } A \leq 0 ;\end{cases}
$$

where

$$
U_{2}\left(P^{m}, q^{*}\left(P^{m}\right)\right)=\left(R-P^{m}-C\left(\frac{\mu \theta+(r+\theta)^{2}}{(r+\theta)\left(r \mu-\lambda q^{*}\left(P^{m}\right)(r+\theta)\right)}\right)\right) q^{*}\left(P^{m}\right)+v\left(1-q^{*}\left(P^{m}\right)\right) .
$$

Proof. The proof of this theorem is given in Appendix A.2. 
Given the characteristics of his system defining the values of $A, B$ and anticipating the strategic behavior of customers depending on the service price, the server manager, playing the role of leader, chooses the price maximizing his utility, according to the relation (5.5). For a service price $P^{*}$ chosen by the server manager, the strategic choice of a customer arriving at the system is described by the relation (5.6).

The results of the Theorem 5.2 can be synthesized as an algorithm, describing the decision-making process of the equilibrium service price to be applied to the server manager (according to the system parameters and the anticipated customer behavior presented in the Lemma 5.1) (see Appendix A.3).

Remark 5.3. The difference that separates our approach on the strategic behavior of agents in queueing systems from the majority of studies that exist on this topic in the literature essentially reduces to the assumption assumed since Naor's work and in the majority of works that deal with strategic behavior in queueing systems. This assumption states that customers are "risk neutral" and we found it in almost all works (see, [12] in Sect. 1.4, page 05).

This assumption that customers are "risk neutral" has a strong influence on the proposed model in general, and more particularly, on the method of its resolution (represented by the indifference principle). Therefore, the researchers working on strategic queueing systems propose to model and solve the game with a non-standard infinite game where the players are all the customers (past and future) and a server manager. This situation consists of two stages, where in the first stage the server manager seeks to maximize his revenue and in the second stage, a game between customers is assumed, where a symmetric equilibrium is found for the game between customers by considering the customers are risk neutral.

In our approach, we assumed that the server manager considers the customers to be homogeneous according to their decision making (i.e. they have the same expected utility function) and they have an optimizer profile (i.e. the customers have an optimizing behavior and are not risk neutral). This leads us to consider that the server manager will adopt "the same behavior" with all the customers that come to his server. The resulting model is a Stackelberg game.

In order to explain the difference between the two approaches (presented in this paper and the so-called standard approach), we give the following numerical example.

For the given parameters of the system $R=40, v=3, C=1, \mu=4, r=3, \theta=1$ and $\lambda=2.5$, we have:

- The Stackelberg equilibrium obtained, by our approach in Theorem 5.2, is

$\left(P^{*}, \alpha^{*}=\left(q^{*}, 1-q^{*}\right)\right)=(31.2471,(0.8771,1229))$.

- The equilibrium obtained, by the standard (classical) approach, will be of the following form:

$\left(P_{S}, \alpha_{S}=\left(q_{S}, 1-q_{S}\right)\right)=\left(R-v-C\left(\frac{\mu \theta+(r+\theta)^{2}}{(r+\theta)(r \mu-\lambda(r+\theta))}\right),(1,0)\right)=(34.5,(1,0))$.

The corresponding server manager and customer utilities are:

$$
\begin{aligned}
& U_{1}\left(P^{*}, q^{*}\right)=68.5134<U_{1}\left(P_{S}, q_{S}\right)=\mathbf{8 6 . 2 6 0 0} \\
& U_{2}\left(P^{*}, q^{*}\right)=\mathbf{6 . 6 8 7 7}>U_{2}\left(P_{S}, q_{S}\right)=3=v .
\end{aligned}
$$

From (5.9) and (5.10), we see that the revenue of the server manager with the equilibrium $\left(P_{S}, \alpha_{S}\right)$ obtained by the classical approach is higher than the revenue of the server manager with the Stackelberg equilibrium $\left(P^{*}, \alpha^{*}\right)$ obtained by our approach. This is justified by the fact that risk neutral customers join the system as soon as their utility is greater than or equal to the outside opportunity. But, if we assume that customers have an optimizer profile, they will choose the mixed strategy $\alpha^{*}=\left(q^{*}, 1-q^{*}\right)$, because it guarantees a higher payoff. Indeed, if an optimizer customer arrives at the server and finds the price $P_{S}$, fixed by the server, then he will choose to join the system with probability $q^{*}\left(P_{S}\right)$ and will receive a profit of $U_{2}\left(P_{S}, q^{*}\left(P_{S}\right)\right)=\mathbf{4 . 0 5 0 5}>3=U_{2}\left(P_{S}, q_{S}\right)$, so he will deviate from the equilibrium strategy $q_{S}$.

Thus, the assumption that customers are risk-neutral must be fully assumed in the standard approach. 


\section{The SOCIAL WelFARE}

The analysis of the strategic behaviors of a server manager and customers has been widely studied in the literature with different modeling approaches. Approaches using game theory tools can be divided into two main families: non-cooperative games in a strategic form (also known as a normal form) (see $[9,10,17,21,22,28,31]$, $\ldots$ ) and non-cooperative games in a dynamic form (see $[6,19,25,27,35], \ldots)$. Most of the works can be integrated into the class of non-cooperative games in strategic form, even if the game models are not always formulated in explicit form. However, in recent years, a number of studies has been carried out to model the strategic interactions of decision makers in queueing systems, which use dynamic game tools (see $[5,6,14,19,27,34,35]$, ...).

Based on the assumption that in reality, a server (rather his manager) behaves as a leader, towards customers, to impose his service price, who are then free to choose to join the system to be served, or to choose an outside opportunity (e.g. a competing server). In this paper, we have modeled these strategic interactions between the server manager and customers in the form of a Stackelberg game, where the server manager is considered as a leader and a potential customer as a follower. In the first part of the paper, we formulated the terms of existence and the explicit form of Stackelberg equilibrium (the equilibrium price for the server manager and the equilibrium joining probability for a potential customer).

In this part, we will study the social welfare problem. Generally, the social planner aims to motivate customers to adopt a socially optimal strategy and the server manager to select an appropriate service price to encourage selfish customers to behave in a socially optimal way. Naor [21] showed that when customers can see the queue before joining it, the server will charge a higher price than the socially optimizer. Since this work, the authors have focused on whether the queueing systems with strategic customers are socially efficient. Edelson and Hildebrand [10] showed that when customers make their decisions without observing the queue, the equilibrium price maximizes social welfare. Afterward, Chen and Frank [6] showed the equivalence between the price displayed by the server and the maximum of the social welfare if the waiting cost is linear. Otherwise, this equivalence does not hold in general. Recently, Wang and Zhang [27] studied how the social planner determines the price to charge a joining customer in the $M / M / 1$ retrial queue with delayed vacations.

In this section, we follow the same principle of reasoning as the first part of the paper, to define the function of the social welfare of the system. Indeed, the question that arises in the system we are dealing with is as follows: is it more favorable for the server manager, acting as a leader, to choose a Stackelberg equilibrium service price, taking into account his own interests as a priority and at the same time imposing non-cooperative behavior of customers or to choose a socially optimal price, taking into account his individual interests and those of his customers.

Thus, in the specific context of our modeling, the social planner aims to motivate the server manager to adopt a socially optimal price and the customers to select an appropriate strategy.

To do this, we adapt below the definition of the social welfare function of the system, for which the decision variable will be the price that the leader will have to choose. Then, we find the socially optimal price that maximizes social welfare, which we compare to the Stackelberg equilibrium already established in Section 5.

Recall that the social welfare function is defined as the sum of the surplus of the server manager and the customers. However, the server surplus $\mathrm{SS}(P)$ is represented by his expected revenue and the customers surplus $\mathrm{CS}(P)$ is represented by the sum of their utilities, given respectively by:

$$
\mathrm{SS}(P)=\lambda q P,
$$

and

$$
\operatorname{CS}(P)=\lambda\left(\left(R-P-C\left(\frac{\mu \theta+(r+\theta)^{2}}{(r+\theta)(r \mu-\lambda q(r+\theta))}\right)\right) q+v(1-q)\right) .
$$

Here, the server manager, acting as a social planner, makes the first move, choosing the service price that maximizes the social welfare function. Therefore, customers play their best response against the social price chosen by the server manager. 
Having defined the strategic behavior of the customers $\mathrm{BR}_{C}(P)=q^{*}(P)$ given by (5.2) and according to the service price $P$ chosen by the server manager, the social planner is expected to resolve the following problem:

$$
\begin{aligned}
\mathrm{SW}(P) & =\operatorname{SS}(P)+\operatorname{CS}(P) \\
& =\lambda q^{*}(P)\left(R-v-C\left(\frac{\mu \theta+(r+\theta)^{2}}{(r+\theta)\left(r \mu-\lambda q^{*}(P)(r+\theta)\right)}\right)\right)+\lambda v \longrightarrow \max _{P \geq 0}
\end{aligned}
$$

i.e. find a socially optimal price:

$$
P_{\mathrm{SW}}^{*} \in \mathcal{P}_{\mathrm{SW}}^{*}=\arg \max _{P \geq 0} \mathrm{SW}(P) .
$$

By substituting $q^{*}(P)$, given by (5.2), in (6.1), the social welfare function $\mathrm{SW}(P)$ takes the following form:

$$
\mathrm{SW}(P)= \begin{cases}\lambda v, & \text { if } P \geq R-v ; \\ \lambda v, & \text { if } P<R-v, B \leq 0 ; \\ \lambda\left(R-C\left(\frac{\mu \theta+(r+\theta)^{2}}{(r+\theta)(r \mu-\lambda(r+\theta))}\right)\right), & \text { if } P<R-v, B>0, A \geq 0, P \in[0, A] \\ \widetilde{\mathrm{SW}}(P), & \text { if } P<R-v, B>0, A \geq 0, P \in[A, B] ; \\ \widetilde{\mathrm{SW}}(P), & \text { if } P<R-v, B>0, A \leq 0, P \in[0, B] \\ \lambda v, & \text { if } P<R-v, B>0, P \geq B,\end{cases}
$$

where $\widetilde{\mathrm{SW}}(P)=\lambda \widetilde{q}(P)\left(R-v-C\left(\frac{\mu \theta+(r+\theta)^{2}}{(r+\theta)(r \mu-\lambda \widetilde{q}(P)(r+\theta))}\right)\right)+\lambda v$ and $\widetilde{q}(P)=\frac{1}{\lambda}\left(\frac{\mu r}{r+\theta}-\sqrt{\frac{C \mu r\left(\mu \theta+(r+\theta)^{2}\right)}{(r+\theta)^{3}(R-P-v)}}\right)$.

Theorem 6.1. In the totally unobservable $M / M / 1$ queue with an unreliable server, the set $\mathcal{P}_{\mathrm{SW}}^{*}$ of socially optimal solutions of the social welfare problem (6.1) is the set of $P_{\mathrm{SW}}^{*}$, given by:

$$
\mathcal{P}_{\mathrm{SW}}^{*}=\arg \max _{P \geq 0} \operatorname{SW}(P)= \begin{cases}{[0,+\infty),} & \text { if } B \leq 0 \\ {[0, A],} & \text { if } B>0 \text { and } A \geq 0 \\ \{0\}, & \text { if } B>0 \text { and } A \leq 0\end{cases}
$$

The socially optimal joining probability is given as follows:

$$
q_{\mathrm{SW}}^{*}= \begin{cases}0, & \text { if } B \leq 0 ; \\ 1, & \text { if } B>0 \text { and } A \geq 0 \\ q_{\mathrm{SW}}=\frac{1}{\lambda}\left(\frac{\mu r}{r+\theta}-\sqrt{\frac{C \mu r\left(\mu \theta+(r+\theta)^{2}\right)}{(r+\theta)^{3}(R-v)}}\right), & \text { if } B>0 \text { and } A \leq 0\end{cases}
$$

The optimal value $\mathrm{SW}^{*}$ of the social welfare is:

$$
\mathrm{SW}^{*}= \begin{cases}\lambda v, & \text { if } B \leq 0 \\ \lambda\left(R-C\left(\frac{\mu \theta+(r+\theta)^{2}}{(r+\theta)(r \mu-\lambda(r+\theta))}\right)\right), & \text { if } B>0 \text { and } A \geq 0 \\ \lambda q_{\mathrm{SW}}\left(R-v-C\left(\frac{\mu \theta+(r+\theta)^{2}}{(r+\theta)\left(r \mu-\lambda q_{\mathrm{SW}}(r+\theta)\right)}\right)\right)+\lambda v, & \text { if } B>0 \text { and } A \leq 0\end{cases}
$$

Proof. The proof of this theorem is presented in Appendix A.4.

The Theorem 6.1 gives us clearly the socially optimal price for different cases with the values of $A$ and $B$. These results are synthesized as an Algorithm, describing the decision-making process of the social service price to be applied to the server manager. (see Appendix A.5). 
After having obtained the Stackelberg equilibrium price $P^{*}$, given by (5.5) and the socially optimal price $P_{\mathrm{SW}}^{*}$ given by (6.3), the server manager, as a leader, must choose between the equilibrium price and the social price it must apply to price his services. If its utility function is his only decision criterion, then the following Theorem gives the price to be chosen according to the parameters of the system.

Theorem 6.2. In the totally unobservable $M / M / 1$ queue with an unreliable server, the server manager is indifferent to choose between the Stackelberg equilibrium price $P^{*}$ and the socially optimal price $P_{\mathrm{SW}}^{*}$ in these cases:

$-B \leq 0$,

$-B>0$ and $A \geq 0$, if $0 \leq P^{m} \leq A$ and $P_{\mathrm{SW}}^{*}=A$.

Otherwise, the server manager has no interest to choose the socially optimal price $P_{\mathrm{SW}}^{*}$ in these cases:

- $B>0$ and $A \geq 0$, if $0 \leq P^{m} \leq A$ and $\forall P_{\mathrm{SW}}^{*} \in[0, A)$,

- $B>0$ and $A \geq 0$, if $A<P^{m} \leq B$ and $\forall P_{\mathrm{SW}}^{*} \in[0, A]$,

- $B>0$ and $A \leq 0$.

Proof. The proof of this theorem appears in Appendix A.6.

\section{Numerical apPlication}

In this section, we present numerical examples that illustrate the effects of some system parameters on the equilibrium strategies obtained for the customers and the server manager, i.e. the equilibrium joining probability of customers and the service price, while ensuring that the stability condition $\mu r>\lambda(r+\theta)$ is verified.

We first studied numerically the effects and the influence of the service price on the joining probability into the system.

Figure 3 represents the variations of the joining probability according to the price $P$, with $R=40, v=3$, $C=1, \mu=4, r=3, \theta=1$ and $\lambda=2.5$. In this figure, we observe that the joining probability to the system equal to 1 when the service price is less than or equal to the acquisition price of all customers $A$. On the other hand, the joining probability decreases as soon as the service price exceeds the price $A$, until it annulled for a price $P \geq B$. Which means that when $P \geq B$, all customers choose the outside opportunity.

The customers behavior is logical. All customers decide to join the system for prices less than or equal to $A$, and a proportion of customers decides to choose the outside opportunity when the service price exceeds $A$. Therefore, the number of customers who join the system increases with the decreasing of the service price and the number of customers who choose the outside opportunity increases with the increase of the service price. Moreover, in the same figure, we can observe that the server manager will choose a service price $P^{*}=P^{m}$ which maximizes his revenue. For a service price $P^{*}=P^{m}$, we note that a proportion of customers decides to join the system and the others decide on the outside opportunity. In the case, where $P^{*}=A$, all customers decide to join the system.

In Figures 4 and 5, we study the variations of the joining probability as a function of service price for different values of $r$ and $\theta$ respectively, in order to clarify the influence of the reliability parameters on the service price. Figure 4 shows that an increase in the repair rate $r$ results an increase in the joining probability and the value of the equilibrium service price. So, for example, for $r=3$, the acquisition price of all customers $A$ equals to 22 , but increasing $r$ to 6 , we get a price $A=32.70$ which is higher. On the other hand, through the Figure 5 where the failure rate $\theta$ is varied, the increase of $\theta$ causes a decrease in the joining probability to the system and the value of the equilibrium service price. When customers arrive at the system, they observe the parameters of the system, so for a high repair rate $r$ customers decide to join the system because the average repair time is very low, but when the failure rate $\theta$ is high, the number of failures is also high, which discourages a proportion of customers and decides on the outside opportunity, even if the repair rate is high. 


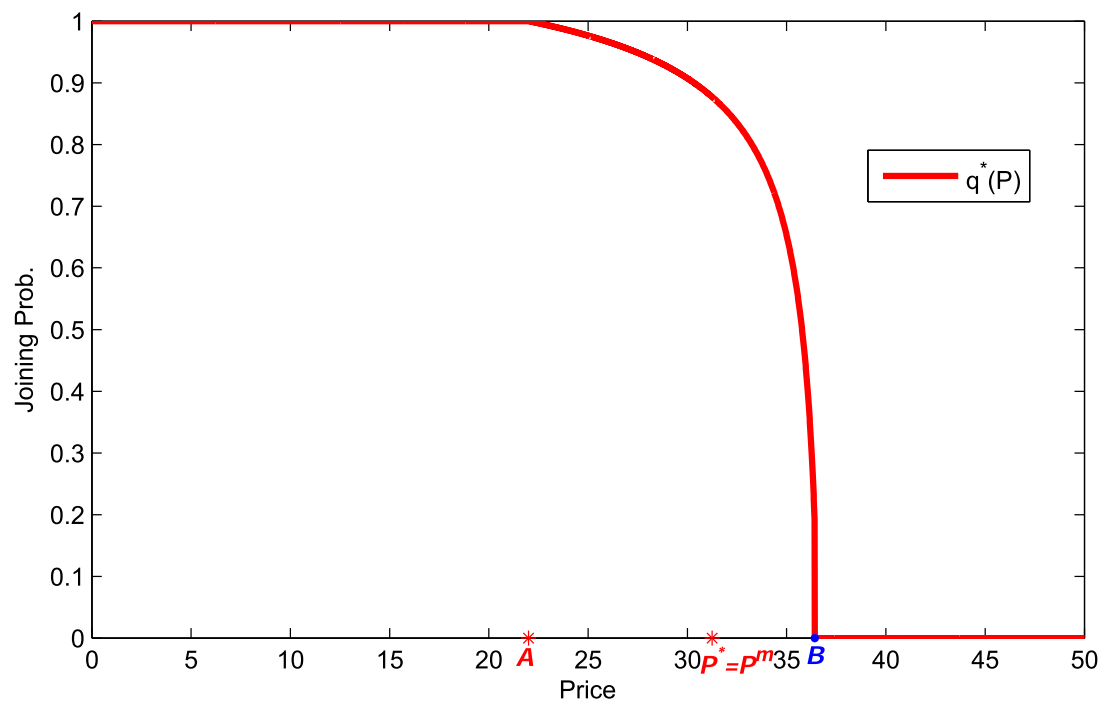

Figure 3. Joining probability vs. Price for $R=40, v=3, C=1, \mu=4, r=3, \theta=1$ and $\lambda=2.5$.

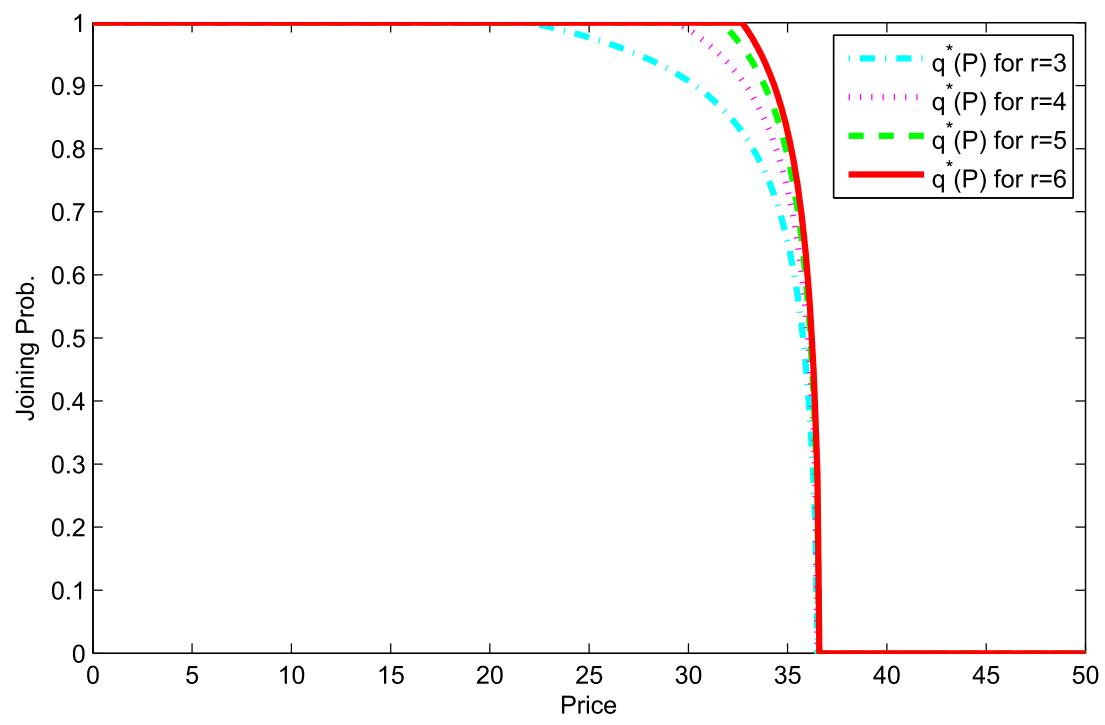

Figure 4. Joining probabilities vs. Price for $R=40, v=3, C=1, \mu=4, \theta=1$ and $\lambda=2.5$.

Figures 6 and 7 show the server's manager revenue based on the service price in the case $B>0$ and $A \geq 0$, and Figure 8 in the case $B>0$ and $A \leq 0$. Figure 6 presents the server's manager revenue with the parameters $R=40, v=3, C=1, \mu=4, r=3, \theta=1$ and $\lambda=2.5$, and Figure 7 shows the server's manager revenue with the parameters $R=30, v=1, C=1, \mu=3, r=2, \theta=1$ and $\lambda=1$. In this figures, we observe that the server's manager revenue is represented by a straight line for all $P \leq A$ and by a concave curve for $P>A$. The optimal price $P^{*}$ that the server manager must choose is $P^{m}$ for the Figure 6 and $A$ for the Figure 7 . Therefore, there 


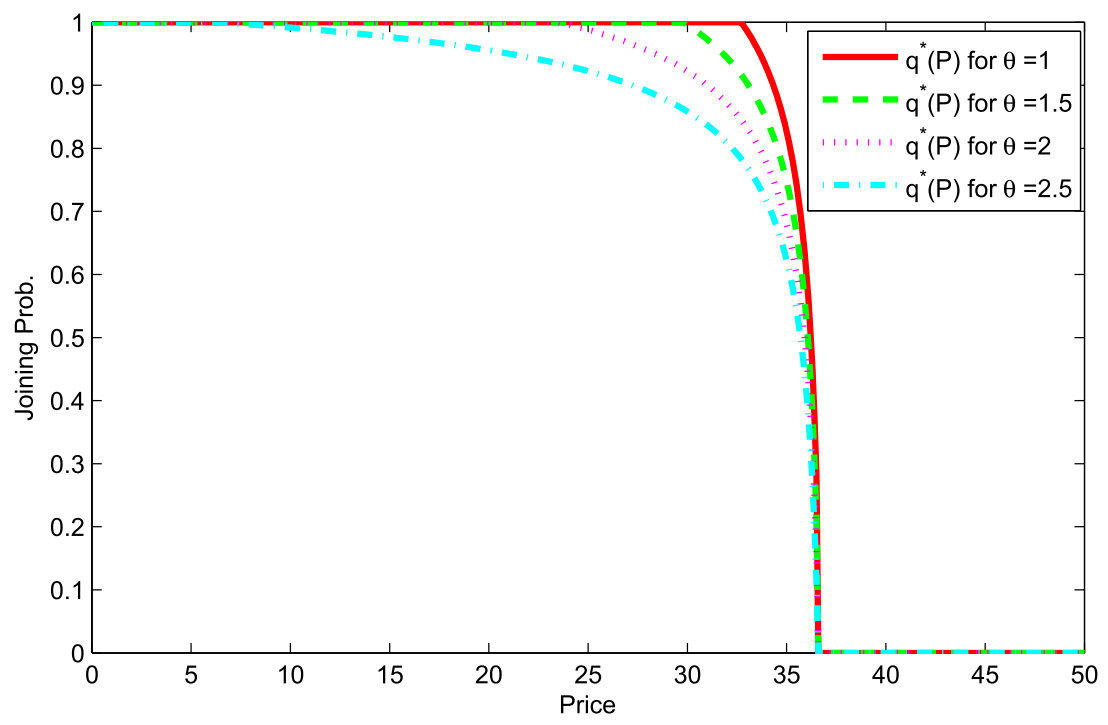

Figure 5. Joining probabilities vs. Price for $R=40, v=3, C=1, \mu=4, r=6$ and $\lambda=2.5$.

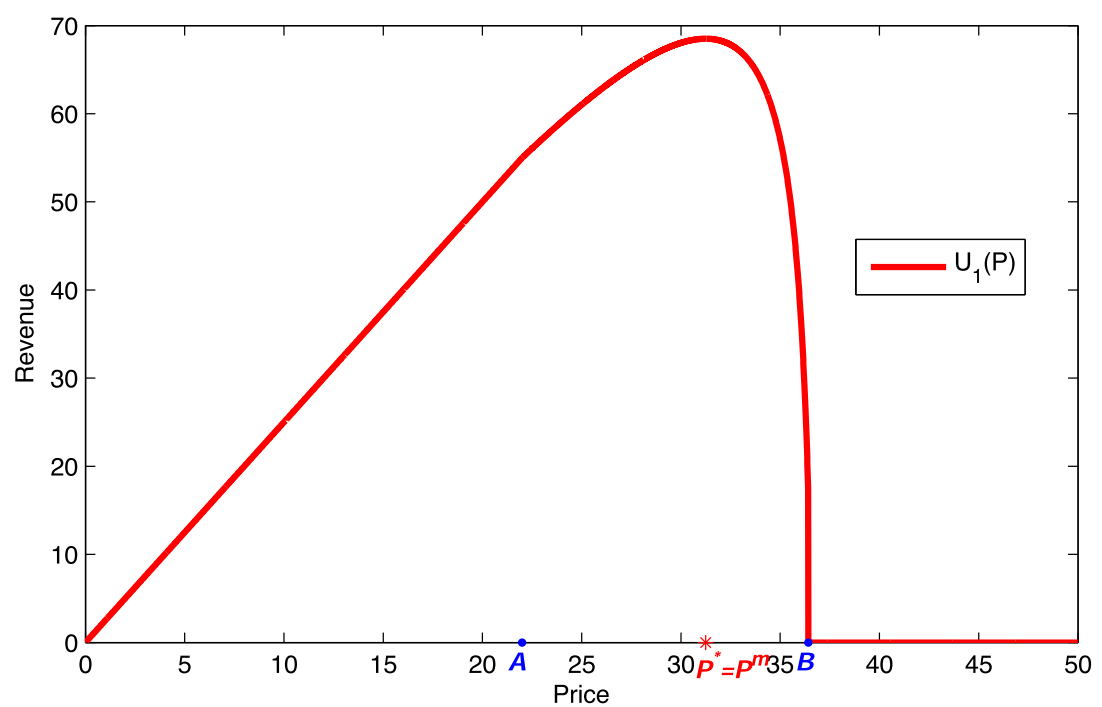

Figure 6. $U_{1}(P)$ vs. Price for $R=40, v=3, C=1, \mu=4, r=3, \theta=1$ and $\lambda=2.5$.

is only a proportion of customers who will decide to join the system if $P^{*}=P^{m}$ (see Fig. 3) and all customers decide to join the system if $P^{*}=A$. Figure 8 represents the server's manager revenue in the case $B>0$ and $A \leq 0$ with the parameters $R=30, v=5, C=2, \mu=4, r=3, \theta=1$ and $\lambda=2.5$. In this figure, we see that the server's manager revenue is represented by a concave curve and the optimal price $P^{*}$ that the server manager must choose is $P^{m}$, because it is the price that gives the maximum revenue. 


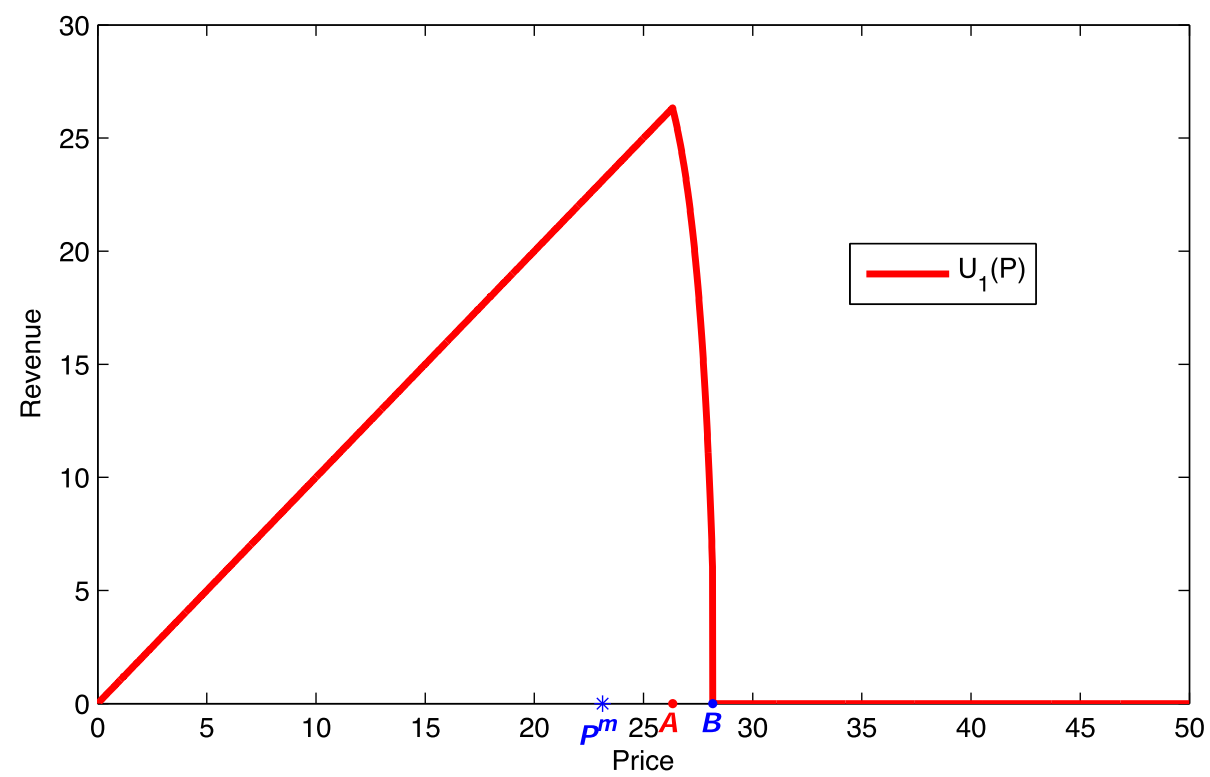

Figure 7. $U_{1}(P)$ vs. Price for $R=30, v=1, C=1, \mu=3, r=2, \theta=1$ and $\lambda=1$.

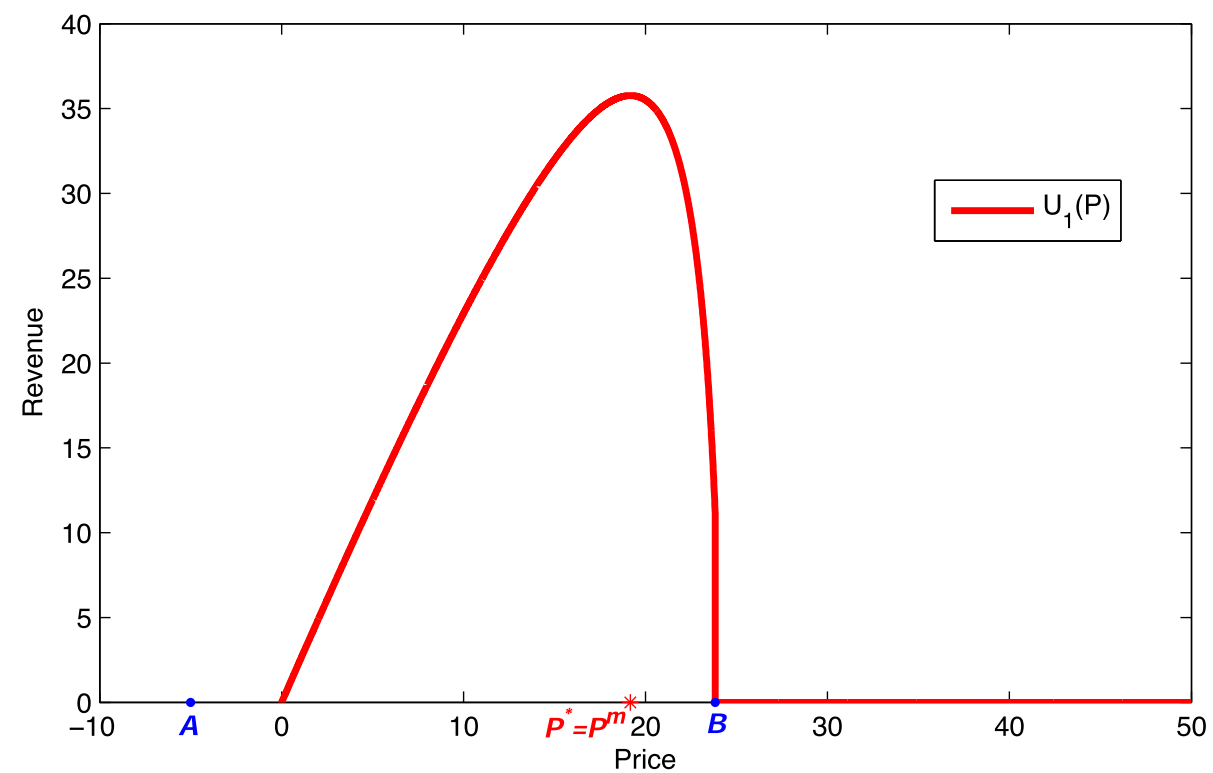

Figure 8. $U_{1}(P)$ vs. Price for $R=30, v=5, C=2, \mu=4, r=3, \theta=1$ and $\lambda=2.5$.

\section{Conclusion}

In this paper, we studied the interactions between the strategic behaviors of the server manager and the customers in an $M / M / 1$ queueing system with unreliable server where the state of the system is totally unobservable. This situation was modeled as Stackelberg game. Then, we determined the equilibrium strategies for the customers and the server manager, namely: the joining probability and the service price at the equilibrium. 
We also studied the social welfare problem and determined the socially optimal price that maximizes the social welfare. We showed that, for the server manager, the Stackelberg equilibrium price does not always coincide with the socially optimal price. The sensitivity of the server manager revenue and the equilibrium joining probability of customers with respect to the service price and some main parameters of the system are explored via numerical experiments. These numerical results can give an idea to the service manager about the policies to be implemented to insure the proper system functioning (economic, service, breakdowns and repairs parameters), while satisfying the customers and the server manager (maximizing their utilities).

There are still many questions and extensions that can be examined for future works. For example, studying the strategic behavior of agents (customers, server manager, social optimizer) in another types of information, such as the totally observable case, the almost observable case, and the almost unobservable case. Then, make a comparison between these types of information, in order to identify the optimal policy to be followed by the manager or the service provider (i.e. reveal information about the length of the queue or not, reveal information about the state of the server or not, ... etc). This study can be also generalized in several directions. One possible direction is to study service pricing and strategic behavior of agents (customers, servers, social optimizer) in the semi-Markovian or in the non-Markovian queueing systems with one or more servers subject to random failures.

\section{ApPENDixes}

\section{Appendix A.1.}

Recall that the customer's objective is to maximize its utility, i.e. to solve the problem:

$$
U_{2}(P, q) \longrightarrow \max _{q \in[0,1]} .
$$

To solve the problem (A.1), we calculate the first and the second derivatives of the utility function, which are given by:

$$
\begin{aligned}
\frac{\partial U_{2}(P, q)}{\partial q} & =R-P-v-C\left(\frac{\mu r\left(\mu \theta+(r+\theta)^{2}\right)}{(r+\theta)(\mu r-\lambda q(r+\theta))^{2}}\right) \\
\frac{\partial^{2} U_{2}(P, q)}{\partial q^{2}} & =-2 \lambda C\left(\frac{\mu r\left(\mu \theta+(r+\theta)^{2}\right)}{(\mu r-\lambda q(r+\theta))^{3}}\right) .
\end{aligned}
$$

From (A.3), and taking into account the stability condition, we deduce:

$$
\frac{\partial^{2} U_{2}(P, q)}{\partial q^{2}}<0, \quad \forall q \in[0,1]
$$

which allows us to conclude that $U_{2}$ is strictly concave on $[0,1]$ and it can only admit one maximum point in the interval $[0,1]$.

We are looking for the solution of the problem (A.1) according to the price $P$ set by the server manager. For this, we will distinguish the following possible cases:

I. If $P \geq R-v$, then $\frac{\partial U_{2}(P, q)}{\partial q}<0, \forall q \in[0,1]$, and the best customer response takes the value:

$$
\mathrm{BR}_{C}(P)=\arg \max _{0 \leq q \leq 1} U_{2}(P, q)=0,
$$

i.e. that the customer chooses with certainty the outside opportunity.

II. If $P<R-v$, we can distinguish different possible cases:

Case 1. $B \leq 0$.

Since

$$
R-v-C\left(\frac{\mu \theta+(r+\theta)^{2}}{(r+\theta)(r \mu-\lambda q(r+\theta))}\right) \leq R-v-C\left(\frac{\mu \theta+(r+\theta)^{2}}{r \mu(r+\theta)}\right), \forall q \in[0,1],
$$


we deduce that:

$$
R-P-v-C\left(\frac{\mu \theta+(r+\theta)^{2}}{(r+\theta)(r \mu-\lambda q(r+\theta)}\right) \leq B \leq 0, \quad \forall q \in[0,1] .
$$

Then, for all $q \in[0,1]$, we have:

$$
U_{2}(P, q)=\left(R-P-C\left(\frac{\mu \theta+(r+\theta)^{2}}{(r+\theta)(r \mu-\lambda q(r+\theta))}\right)\right) q+v(1-q) \leq v=U_{2}\left(P, q^{*}(P)=0\right) .
$$

Hence, the best customer response $\mathrm{BR}_{C}(P)$ is:

$$
\mathrm{BR}_{C}(P)=\arg \max _{0 \leq q \leq 1} U_{2}(P, q)=0 .
$$

Case 2. $B>0$ and $A \geq 0$.

Case 2.1. $0 \leq P \leq A$.

Since

$$
\frac{\partial U_{2}(P, q)}{\partial q}=R-P-v-C\left(\frac{\mu r\left(\mu \theta+(r+\theta)^{2}\right)}{(r+\theta)(\mu r-\lambda q(r+\theta))^{2}}\right) \geq A-P \geq 0, \forall q \in[0,1],
$$

we deduce that the function $U_{2}$ is increasing in $q$ on $[0,1]$ and reaches its maximum at the point $q^{*}(P)=1$. Thus, the best customer response $\mathrm{BR}_{C}(P)$ is:

$$
\operatorname{BR}_{C}(P)=\arg \max _{0 \leq q \leq 1} U_{2}(P, q)=1 .
$$

Case 2.2. $A \leq P \leq B$.

The necessary condition of the first order $\frac{\partial U_{2}(P, q)}{\partial q}=0$, gives us two possible solutions:

$$
q_{1}(P)=\frac{1}{\lambda}\left(\frac{\mu r}{r+\theta}-\sqrt{\frac{C \mu r\left(\mu \theta+(r+\theta)^{2}\right)}{(r+\theta)^{3}(R-P-v)}}\right)
$$

and

$$
q_{2}(P)=\frac{1}{\lambda}\left(\frac{\mu r}{r+\theta}+\sqrt{\frac{C \mu r\left(\mu \theta+(r+\theta)^{2}\right)}{(r+\theta)^{3}(R-P-v)}}\right) .
$$

The second solution (A.6) is rejected because $\mu r>\lambda(r+\theta)$ and therefore $q_{2}(P)>1$. On the other hand, it is easy to verify that the solution $q_{1}(P)$, given by (A.5), belongs to the interval $[0,1]$.

Therefore, whatever the price $P \in[A, B]$, chosen by the server manager, the best customer response will be:

$$
\operatorname{BR}_{C}(P)=\frac{1}{\lambda}\left(\frac{\mu r}{r+\theta}-\sqrt{\frac{C \mu r\left(\mu \theta+(r+\theta)^{2}\right)}{(r+\theta)^{3}(R-P-v)}}\right) .
$$

Case 3. $B>0, A \leq 0$ and $0 \leq P \leq B$.

With an analogous reasoning of the Case 2.2, we deduce that the solution (A.6) will be rejected, and the solution (A.5) belongs to the interval $[0,1]$.

Hence, the best customer response $\operatorname{BR}_{C}(P)$ facing any price $P \in[0, B]$, takes the form (A.7).

Case 4. $B>0$ and $P \geq B$.

For $q \in[0,1]$, we have:

$$
U_{2}(P, q)=\left(R-v-C\left(\frac{\mu \theta+(r+\theta)^{2}}{(r+\theta)(r \mu-\lambda q(r+\theta))}\right)\right) q-P q+v .
$$


Since

$$
\frac{\mu \theta+(r+\theta)^{2}}{(r+\theta)(r \mu-\lambda q(r+\theta))} \geq \frac{\mu \theta+(r+\theta)^{2}}{(r+\theta) r \mu}, \forall q \in[0,1]
$$

then:

$$
U_{2}(P, q) \leq B q-P q+v=(B-P) q+v \leq v=U_{2}(P, q=0), \forall q \in[0,1] .
$$

Hence, the best customer response is: $\operatorname{BR}_{C}(P)=0$.

The analysis of the different possible cases (see Cases I-II-4) shows that there is, in each of them, only one single stationary point of the function $U_{2}$ in the interval $[0,1]$, which, taking into account (A.4), will be the unique solution of the problem (A.1) for each price $P$ fixed by the server.

\section{Appendix A.2.}

I. If $B \leq 0$, according to (5.3), we have $U_{1}(P)=0, \forall P \in[0,+\infty)$, then

$$
P^{*} \in \arg \max _{P \in[0,+\infty)} U_{1}(P)=[0,+\infty) .
$$

II. If $B>0$ and $A \geq 0$.

II.1. For $P \in[0, A]$.

In this case, according to (5.3), the expected utility function of the server manager takes the form:

$$
U_{1}(P)=\lambda P
$$

which is linear with respect of $P$, reaches, then, its maximum at the point $P^{*}=A$, i.e.

$$
P^{*}=\arg \max _{P \in[0, A]} U_{1}(P)=A=R-v-C\left(\frac{\mu r\left(\mu \theta+(r+\theta)^{2}\right)}{(r+\theta)(r \mu-\lambda(r+\theta))^{2}}\right) .
$$

Hence,

$$
\max _{0 \leq P \leq A} U_{1}(P)=U_{1}(A)=\lambda\left(R-v-C\left(\frac{\mu r\left(\mu \theta+(r+\theta)^{2}\right)}{(r+\theta)(r \mu-\lambda(r+\theta))^{2}}\right)\right) .
$$

II.2. For $P \in[A, B]$.

According to (5.3), the utility of the server manager is written:

$$
U_{1}(P)=\left(\frac{\mu r}{r+\theta}-\sqrt{\frac{C \mu r\left(\mu \theta+(r+\theta)^{2}\right)}{(r+\theta)^{3}(R-P-v)}}\right) P .
$$

We have:

$$
\frac{\partial U_{1}(P)}{\partial P}=\frac{\mu r}{r+\theta}+\frac{(-2 R+P+2 v) \sqrt{\frac{C \mu r\left(\mu \theta+(r+\theta)^{2}\right)}{(r+\theta)^{3}(R-P-v)}}}{2(R-P-v)} .
$$

The necessary condition of optimality gives us only one feasible solution:

$$
P^{m}=R-v-F .
$$

Since

$$
\frac{\partial^{2} U_{1}(P)}{\partial P^{2}}=\frac{(-4 R+P+4 v) \sqrt{\frac{C \mu r\left(\mu \theta+(r+\theta)^{2}\right)}{(r+\theta)^{3}(R-P-v)}}}{4(R-P-v)^{2}}<0,
$$

then the function $U_{1}(P)$ is strictly concave and $P^{m}$ is the maximum point. 
- If $A \leq P^{m} \leq B$, then the maximum point of $U_{1}$ in $[A, B]$ is reached at the point $P^{m}$ defined in (A.10)

- If $P^{m} \leq A$, we have $\frac{\partial U_{1}(P)}{\partial P}<0$ in $\left[P^{m}, B\right]$, and the maximum point of $U_{1}$ in $[A, B]$ is reached at the point $A$.

Hence,

$$
\begin{gathered}
P^{*}=\arg \max _{P \in[A, B]} U_{1}(P)= \begin{cases}P^{m}, & \text { if } A \leq P^{m} \leq B \\
A, & \text { if } P^{m} \leq A .\end{cases} \\
\max _{A \leq P \leq B} U_{1}(P)=U_{1}\left(P^{*}\right)=\left(\frac{\mu r}{r+\theta}-\sqrt{\frac{C \mu r\left(\mu \theta+(r+\theta)^{2}\right)}{(r+\theta)^{3}\left(R-P^{*}-v\right)}}\right) P^{*} .
\end{gathered}
$$

III. If $B>0$ and $A \leq 0$. For $P \in[0, B]$.

According to (5.3), the utility function $U_{1}(P)$ of the server manager takes the form (A.8), and the necessary condition of optimality gives one feasible solution $P^{m}$ defined by (A.10).

Since $U_{1}(0)=U_{1}(B)=0$ and from $(\mathrm{A} .9), \frac{\partial^{2} U_{1}(P)}{\partial P^{2}}<0$, then the function $U_{1}(P)$ is strictly concave on $[0, B]$, hence $P^{m} \in[0, B]$.

Therefore,

$$
P^{*}=\arg \max _{P \in[0, B]} U_{1}(P)=P^{m},
$$

and

$$
\max _{0 \leq P \leq B} U_{1}(P)=U_{1}\left(P^{m}\right)=\left(\frac{\mu r}{r+\theta}-\sqrt{\frac{C \mu r\left(\mu \theta+(r+\theta)^{2}\right)}{(r+\theta)^{3}\left(R-P^{m}-v\right)}}\right) P^{m} .
$$

\section{Appendix A.3.}

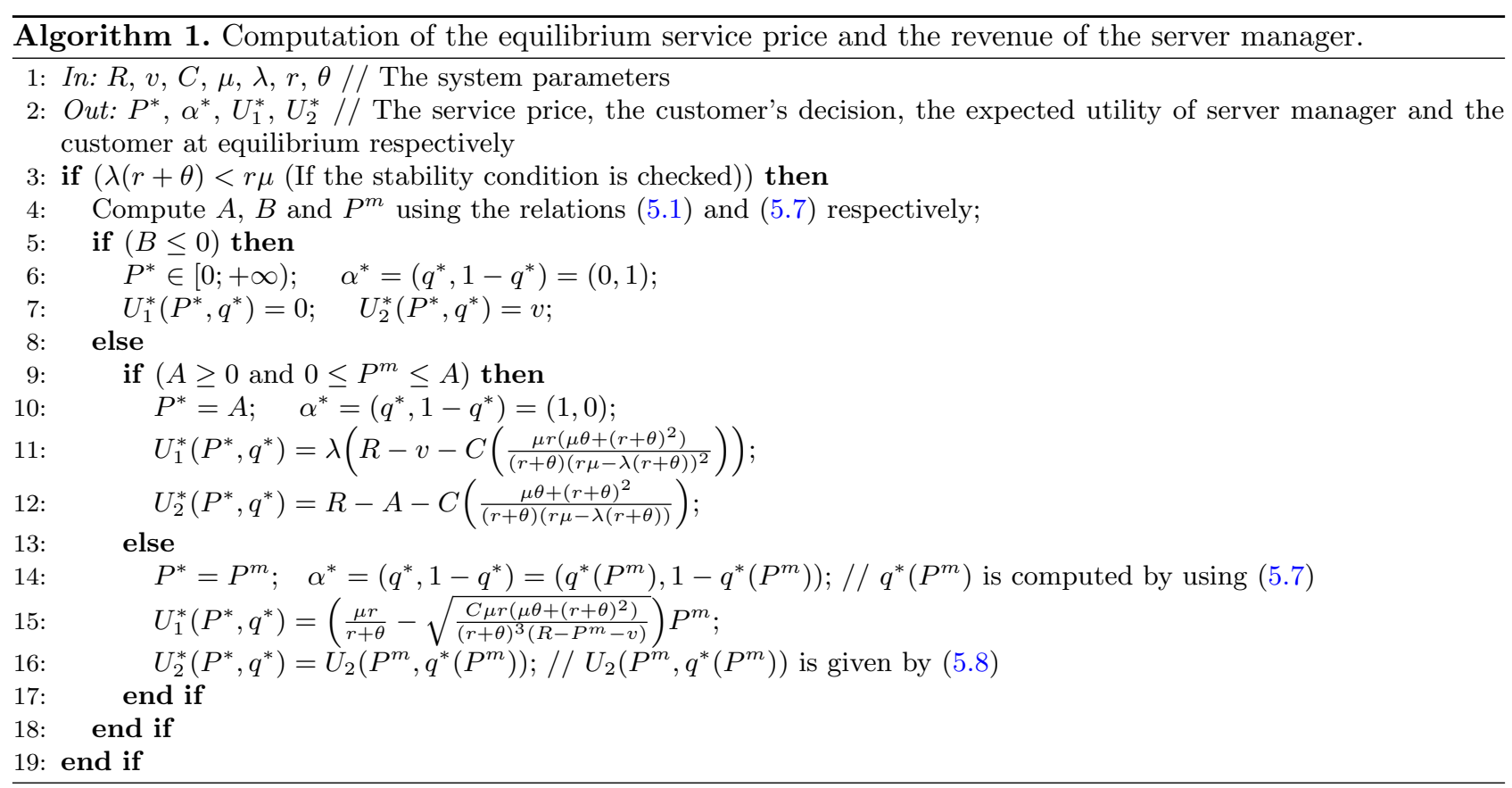




\section{Appendix A.4.}

I. If $B \leq 0$, according to $(6.2)$, the function $\mathrm{SW}(P)$ does not depend on $P$, then:

$$
\mathcal{P}_{\mathrm{SW}}^{*}=\arg \max _{P \in[0,+\infty)} \mathrm{SW}(P)=[0,+\infty), \quad \text { and } \quad \mathrm{SW}^{*}=\max _{P \geq 0} \mathrm{SW}(P)=\lambda v .
$$

II. If $B>0$ and $A \geq 0$. In this case, we have two sub-cases:

- For $P \in[0, A]$.

According to (6.2), we have $\mathrm{SW}(P)=\lambda\left(R-C\left(\frac{\mu \theta+(r+\theta)^{2}}{(r+\theta)(r \mu-\lambda(r+\theta))}\right)\right), \forall P \in[0, A]$. Since the function SW does not depend on $P$, then:

$$
\mathcal{P}_{\mathrm{SW}}^{*}=\arg \max _{P \in[0, A]} \mathrm{SW}(P)=[0, A] .
$$

Hence:

$$
\mathrm{SW}^{*}=\max _{0 \leq P \leq A} \mathrm{SW}(P)=\lambda\left(R-C\left(\frac{\mu \theta+(r+\theta)^{2}}{(r+\theta)(r \mu-\lambda(r+\theta))}\right)\right) .
$$

- For $P \in[A, B]$.

According to (6.2), the social welfare function takes the form:

$$
\mathrm{SW}(P)=\lambda \widetilde{q}(P)\left(R-v-C\left(\frac{\mu \theta+(r+\theta)^{2}}{(r+\theta)(r \mu-\lambda \widetilde{q}(P)(r+\theta))}\right)\right)+\lambda v,
$$

where $\widetilde{q}(P)=\frac{1}{\lambda}\left(\frac{\mu r}{r+\theta}-\sqrt{\frac{C \mu r\left(\mu \theta+(r+\theta)^{2}\right)}{(r+\theta)^{3}(R-P-v)}}\right)$.

To solve the problem (6.1) we calculate the first derivative of the social welfare function (A.11), which is given by:

$$
\frac{\partial \mathrm{SW}(P)}{\partial P}=\frac{P \sqrt{\frac{C \mu r\left(\mu \theta+(r+\theta)^{2}\right)}{(r+\theta)^{3}(R-P-v)}}}{2(P-R+v)} .
$$

We have $\frac{\partial \mathrm{SW}(P)}{\partial P}<0, \forall P \in[A, B]$ and the maximum point of $\mathrm{SW}$ in $[A, B]$ is reached at the point $A$. Hence:

$$
\mathcal{P}_{\mathrm{SW}}^{*}=\arg \max _{P \in[A, B]} \mathrm{SW}(P)=\{A\},
$$

and

$$
\mathrm{SW}^{*}=\max _{A \leq P \leq B} \mathrm{SW}(P)=\mathrm{SW}(A)=\lambda\left(R-C\left(\frac{\mu \theta+(r+\theta)^{2}}{(r+\theta)(r \mu-\lambda(r+\theta))}\right)\right) .
$$

III. If $B>0$ and $A \leq 0$. For $P \in[0, B]$.

In this case, according to (6.2), the social welfare function takes the form (A.11), its first derivative of the form (A.12).

Since $\frac{\partial \mathrm{SW}(P)}{\partial P}<0, \forall P \in[0, B]$, the maximum point of $\mathrm{SW}$ in $[0, B]$ is reached at 0 . Hence:

$$
\mathcal{P}_{\mathrm{SW}}^{*}=\arg \max _{P \in[0, B]} \mathrm{SW}(P)=\{0\},
$$

and

$$
\mathrm{SW}^{*}=\max _{0 \leq P \leq B} \mathrm{SW}(P)=\mathrm{SW}\left(P_{\mathrm{SW}}^{*}\right)=\lambda q_{\mathrm{SW}}\left(R-v-C\left(\frac{\mu \theta+(r+\theta)^{2}}{(r+\theta)\left(r \mu-\lambda q_{\mathrm{SW}}(r+\theta)\right)}\right)\right)+\lambda v,
$$

where $q_{\mathrm{SW}}=\frac{1}{\lambda}\left(\frac{\mu r}{r+\theta}-\sqrt{\frac{C \mu r\left(\mu \theta+(r+\theta)^{2}\right)}{(r+\theta)^{3}(R-v)}}\right)$. 


\section{Appendix A.5.}

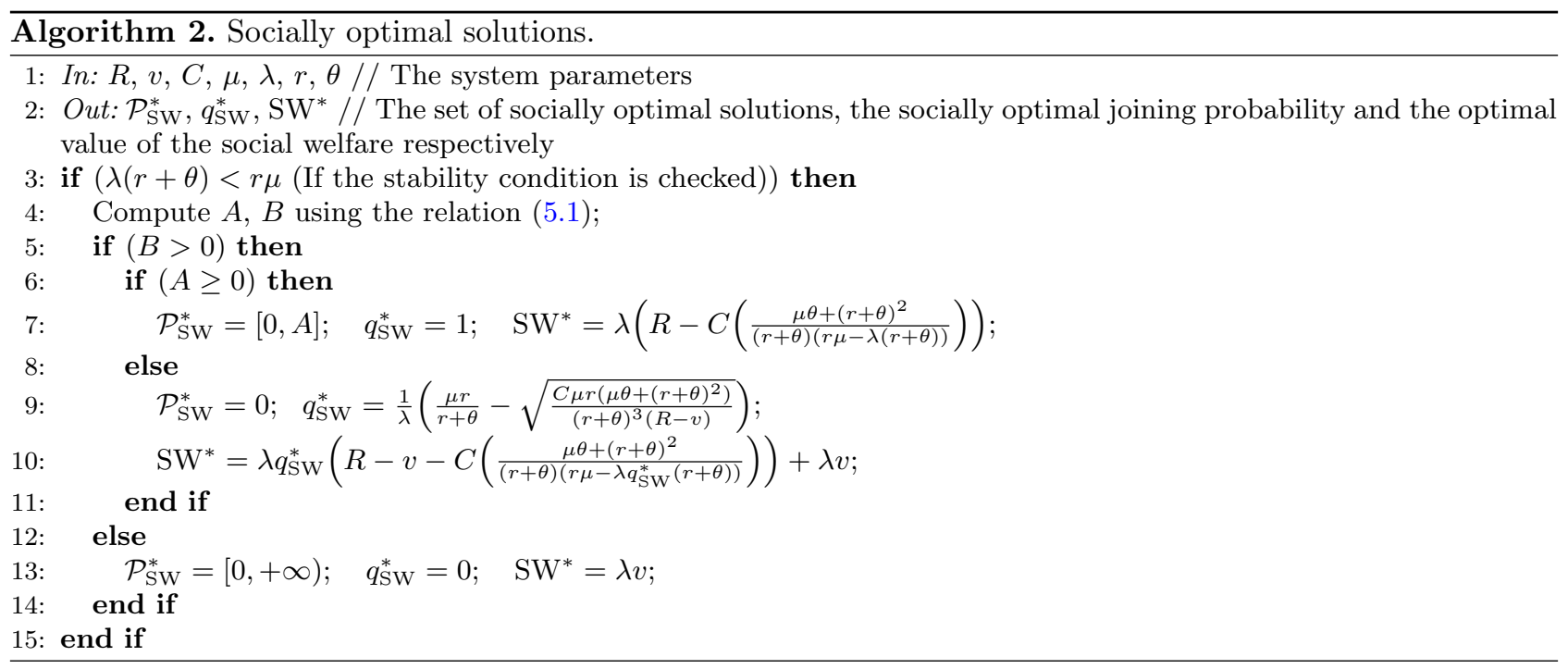

\section{Appendix A.6.}

Recall that the server manager revenue is: $U_{1}(P)=\lambda q P$.

I. If $B \leq 0$, then, $\forall P \in[0, \infty)$, we have: $q^{*}=q_{\mathrm{SW}}^{*}=0$, then:

$$
U_{1}\left(P^{*}\right)=U_{1}\left(P_{\mathrm{SW}}^{*}\right)=0 .
$$

II. If $B>0$ and $A \geq 0$, we will distinguish three cases:

II.1. If $0 \leq P^{m} \leq A$ and $P_{\mathrm{SW}}^{*}=A$, then $P^{*}=P_{\mathrm{SW}}^{*}=A$ and

$$
U_{1}\left(P^{*}\right)=U_{1}\left(P_{\mathrm{SW}}^{*}\right)=\lambda A=\lambda\left(R-v-C\left(\frac{\mu r\left(\mu \theta+(r+\theta)^{2}\right)}{(r+\theta)(r \mu-\lambda(r+\theta))^{2}}\right)\right) .
$$

II.2. If $0 \leq P^{m} \leq A$ and $P_{\mathrm{SW}}^{*} \in[0, A)$, then

$$
U_{1}\left(P^{*}\right)=U_{1}(A)>U_{1}\left(P_{\mathrm{SW}}^{*}\right), \quad \forall P_{\mathrm{SW}}^{*} \in[0, A) .
$$

II.3. If $A<P^{m} \leq B$, then $U_{1}\left(P^{*}\right)=U_{1}\left(P^{m}\right)>U_{1}\left(P_{\mathrm{SW}}^{*}\right), \forall P_{\mathrm{SW}}^{*} \in[0, A]$.

III. If $B>0$ and $A \leq 0$, then $P_{\mathrm{SW}}^{*}=0$ and $U_{1}\left(P^{*}\right)>U_{1}\left(P_{\mathrm{SW}}^{*}\right)=0$.

Acknowledgements. The authors thank the editor and the anonymous reviewers for their remarks, comments, and their valuable suggestions, which have greatly improved the presentation of the paper.

\section{REFERENCES}

[1] A. Aghsami and F. Jolai, Equilibrium threshold strategies and social benefits in the fully observable Markovian queues with partial breakdowns and interruptible setup/closedown policy. Qual. Technol. Quant. Manage. 17 (2020) 685-722.

[2] T. Başar and G.J. Olsder, Dynamic Noncooperative Game Theory. SIAM (1998).

[3] O. Boudali and A. Economou, Optimal and equilibrium balking strategies in the single server Markovian queue with catastrophes. Eur. J. Oper. Res. 218 (2012) 708-715.

[4] O. Boudali and A. Economou, The effect of catastrophes on the strategic customer behavior in queueing systems. Nav. Res. Logistics (NRL) 60 (2013) 571-587. 
[5] H. Chen and M.Z. Frank, State dependent pricing with a queue. IIE Trans. 33 (2001) 847-860.

[6] H. Chen and M. Frank, Monopoly pricing when customers queue. IIE Trans. 36 (2004) 569-581.

[7] P. Chen and Y. Zhou, Equilibrium balking strategies in the single server queue with setup times and breakdowns. Oper. Res. 15 (2015) 213-231.

[8] A. Economou, The impact of information structure on strategic behavior in queueing systems. In: Queueing Theory 2, Advanced Trends. Wiley/ISTE (2021).

[9] A. Economou and S. Kanta, Equilibrium balking strategies in the observable single-server queue with breakdowns and repairs. Oper. Res. Lett. 36 (2008) 696-699.

[10] N.M. Edelson and D.K. Hilderbrand, Congestion tolls for poisson queuing processes. Econ.: J. Econ. Soc. 43 (1975) $81-92$.

[11] S. Gao, D. Zhang, H. Dong and X. Wang, Equilibrium balking strategies in the reparable $M / M / 1 G$-retrial queue with complete removals. Prob. Eng. Inf. Sci. 35 (2021) 138-157.

[12] R. Hassin, Rational Queueing. CRC Press (2016).

[13] R. Hassin and M. Haviv, To Queue or Not to Queue: Equilibrium Behavior in Queueing Systems. Vol. 59. Springer Science \& Business Media (2003)

[14] K. Jagannathan, I. Menache, E. Modiano and G. Zussman, Non-cooperative spectrum accessthe dedicated vs. free spectrum choice. IEEE J. Sel. Areas Commun. 30 (2012) 2251-2261.

[15] K. Li and J. Wang, Equilibrium balking strategies in the single-server retrial queue with constant retrial rate and catastrophes. Qual. Technol. Quant. Manage. 18 (2021) 156-178.

[16] L. Li, J. Wang and F. Zhang, Equilibrium customer strategies in Markovian queues with partial breakdowns. Comput. Ind. Eng. 66 (2013) 751-757.

[17] X. Li, J. Wang and F. Zhang, New results on equilibrium balking strategies in the single-server queue with breakdowns and repairs. Appl. Math. Comput. 241 (2014) 380-388.

[18] Q. Ma and X. Zhang, Analysis and comparison of queue with-policy and unreliable server. Discrete Dyn. Nat. Soc. 2020 (2020) DOI: $10.1155 / 2020 / 6195080$.

[19] A. Manou, P.G. Canbolat and F. Karaesmen, Pricing in a transportation station with strategic customers. Prod. Oper. Manage. 26 (2017) 1632-1645.

[20] K. Meziani, F. Rahmoune and M.S. Radjef, Service pricing and customer behavior strategies of stackelberg's equilibrium in an unobservable Markovian queue with unreliable server and delayed repairs. Int. J. Math. Oper. Res. (2021) DOI: 10.1504/IJMOR.2021.10038485.

[21] P. Naor, The regulation of queue size by levying tolls. Econ.: J. Econ. Soc. 37 (1969) 15-24.

[22] G. Panda, V. Goswami and A.D. Banik, Equilibrium behaviour and social optimization in Markovian queues with impatient customers and variant of working vacations. RAIRO-Oper. Res. 51 (2017) 685-707.

[23] R. Tian, Equilibrium strategies in the almost unobservable queues with catastrophes and repairs. In: 2019 Chinese Control Conference (CCC). IEEE (2019) 1321-1326.

[24] R. Tian and Y. Wang, Analysis of equilibrium strategies in Markovian queues with negative customers and working breakdowns. IEEE Access 7 (2019) 159868-159878.

[25] R. Tian and Y. Wang, Optimal strategies and pricing analysis in $M / M / 1$ queues with a single working vacation and multiple vacations. RAIRO-Oper. Res. 54 (2020) 1593-1612.

[26] J. Wang and F. Zhang, Equilibrium analysis of the observable queues with balking and delayed repairs. Appl. Math. Comput. 218 (2011) 2716-2729.

[27] J. Wang and F. Zhang, Monopoly pricing in a retrial queue with delayed vacations for local area network applications. IMA J. Manage. Math. 27 (2016) 315-334.

[28] S. Yu, Z. Liu and J. Wu, Equilibrium strategies of the unobservable $M / M / 1$ queue with balking and delayed repairs. Appl. Math. Comput. 290 (2016) 56-65.

[29] S. Yu, Z. Liu and J. Wu, Strategic behavior in the partially observable Markovian queues with partial breakdowns. Oper. Res. Lett. 45 (2017) 471-474.

[30] S. Yu, Z. Liu and J. Wu, Optimal balking strategies in the $M / M / 1$ queue with multi-phase failures and repairs. Oper. Res. 19 (2019) 435-447.

[31] Y. Zhang, Strategic behavior in the constant retrial queue with a single vacation. RAIRO-Oper. Res. 54 (2020) $569-583$.

[32] Y. Zhang and J. Wang, Strategic joining and information disclosing in Markovian queues with an unreliable server and working vacations. Qual. Technol. Quant. Manage. 18 (2021) 298-325.

[33] S. Zhang and X. Xu, Equilibrium customer strategies in Markovian queues with setup times and partial failures. J. Syst. Sci. Complexity 33 (2020) 1163-1178.

[34] S. Ziani, F. Rahmoune and M.S. Radjef, Customers' strategic behavior in batch arrivals $M^{2} / M / 1$ queue. Eur. J. Oper. Res. 247 (2015) 895-903.

[35] S. Ziani, F. Rahmoune and M.S. Radjef, Equilibrium behavioural strategies in an $M / M / 1$ queue. Int. J. Math. Oper. Res. 13 (2018) 377-400. 


\section{Subscribe to Open (S2O) A fair and sustainable open access model}

This journal is currently published in open access under a Subscribe-to-Open model (S2O). S2O is a transformative model that aims to move subscription journals to open access. Open access is the free, immediate, online availability of research articles combined with the rights to use these articles fully in the digital environment. We are thankful to our subscribers and sponsors for making it possible to publish this journal in open access, free of charge for authors.

\section{Please help to maintain this journal in open access!}

Check that your library subscribes to the journal, or make a personal donation to the $\mathrm{S} 2 \mathrm{O}$ programme, by contacting subscribers@edpsciences.org

More information, including a list of sponsors and a financial transparency report, available at: https://www. edpsciences.org/en/maths-s2o-programme 